\title{
Contrast and spatial variables in texture segregation: Testing a simple spatial-frequency channels model
}

\author{
ANNE SUTTER and JACOB BECK \\ University of Oregon, Eugene, Oregon \\ and \\ NORMA GRAHAM \\ Columbia University, New York, New York
}

\begin{abstract}
Observers were shown patterns composed of two textures in which each texture contained two types of elements. The elements were arranged in a striped pattern in the top and bottom regions and in a checked pattern in the center region. Observers rated the degree to which the three regions were seen as distinct. When the elements were squares or lines, perceived segregation resulting from differences in element size could be canceled by differences in element contrast. Minimal perceived segregation occurred when the products of the area and the contrast (areal contrasts) of the elements were equal. This dependence of perceived segregation on the areal contrasts of the elements is consistent with a simple model based on the hypothesis that the perceived segregation of the regions is a function of their differential stimulation of spatial-frequency channels. Two aspects of the data were not consistent with quantitative predictions of the model. First, as the size difference between the large and small elements increased, the ratings at the point of minimum perceived segregation increased. Second, some effects of changing the fundamental frequency of the textures were not predicted by the model. These discrepancies may be explained by a more complex model in which a rectification or similar nonlinearity occurs between two stages of orientation- and spatial-frequency-selective linear filters.
\end{abstract}

Models of texture segregation fall into three classes. In one class of models, texture segregation is based on the geometric features of a texture pattern. Beck (1972, 1982) and Marr (1976) proposed that texture segregation is based on differences in first-order statistics of simple features of a texture pattern, such as the slopes and sizes of texture elements or of their component parts. Julesz (1981) has labeled such features textons and proposed that there are three kinds: elongated blobs (e.g., line segments), terminators (e.g., line terminations), and intersections (e.g., crossings of line segments). In a second class of models, the primitives for texture segregation are not geometric features but the outputs of receptive-fieldlike operators. In many of the models in this class, texture segregation is based on differences in image statistics following the convolution of a texture with local, linear filters that have weighting functions like the receptive fields of simple cells. A number of investigators have

This research was supported by AFOSR Grant AFOSR-85-0359 to Jacob Beck and by NIMH Fellowship MH09449 to Norma Graham. Experiments 1 and 4 were performed in partial fulfillment of the requirements for the PhD degree at the University of Oregon by Anne Sutter. Much of this work was presented at the May 1988 meeting of the Association of Researchers in Vision and Ophthalmology (Sutter, Graham, \& Beck, 1988). Correspondence may be addressed to Jacob Beck, Department of Psychology, University of Oregon, Eugene, OR 97403. proposed that texture segregation is, at least sometimes, directly based on differences in the outputs of spatialfrequency channels (e.g., Beck, Sutter, \& Ivry, 1987; Bergen, in press; Bergen \& Adelson, 1988; Bovik, Clark, \& Geisler, 1987; Caelli, 1985; Clark, Bovik, \& Geisler, 1987; Daugman, 1987, 1988; Ginsburg, 1984; Graham, 1981; Grossberg \& Mingolla, 1985; Turner, 1986). Spatial-frequency channels are quasi-independent, parallel channels composed of local receptive fields that are distributed throughout the visual field and are alike in their sensitivity to spatial frequency and orientation. The evidence that the visual system contains a set of spatialfrequency channels, and their usefulness in visual modeling, has been reviewed elsewhere (e.g., Graham, 1980, 1981, 1985).

In a third class of models, texture segregation is based on differences in second-order statistics of the luminances at different points in the texture. The second-order statistics of a region are based on the joint probability distribution that a pair of points separated by a given distance and orientation have particular gray levels. Julesz's (1975) original conjecture considered two gray levels, and the extension of this conjecture to patterns containing many gray levels must be done carefully (Klein \& Tyler, 1986). Julesz (1975) conjectured that textures with the same global second-order statistics do not segregate, but counterexamples to Julesz's conjecture have been found 
(Julesz, Gilbert, \& Victor, 1978; Victor \& Brodie, 1978). Gagalowicz (1981) pointed out that the counterexamples involve patterns in which local second-order statistics are not the same throughout the pattern and differ from the global second-order statistics. He hypothesized that textures that have the same local second-order statistics throughout will fail to segregate. It should be noted that if texture segregation is a function of only the amplitudes, and not the phases, of the spatial frequencies, then the spatial-frequency channel approach is closely related to models based on second-order statistics. Therefore, many of the counterexamples to Julesz's conjecture are also indications that a simple spatial-frequency channels explanation of texture segregation may fail (see Beck, 1983; Julesz \& Caelli, 1979).

Beck et al. (1987) reported results suggesting that perceived texture segregation in certain patterns is determined by differences in the response of spatial-frequency chan-, nels to the different texture regions of the pattern. The patterns they employed were members of a class of patterns investigated by Beck, Prazdny, and Rosenfeld (1983). Figure 1 is an example of this class of patterns. The pattern is periodic, and the three regions in the pattern are composed of approximately equal numbers of two types of elements (dark and light squares in Figure 1). The textures to be segregated differ in the arrangement of the two types of element. In the top and bottom regions, the dark and light squares are arranged in vertical stripes. In the center region, they are arranged in a checked pattern. When viewing the pattern, subjects reported spon-

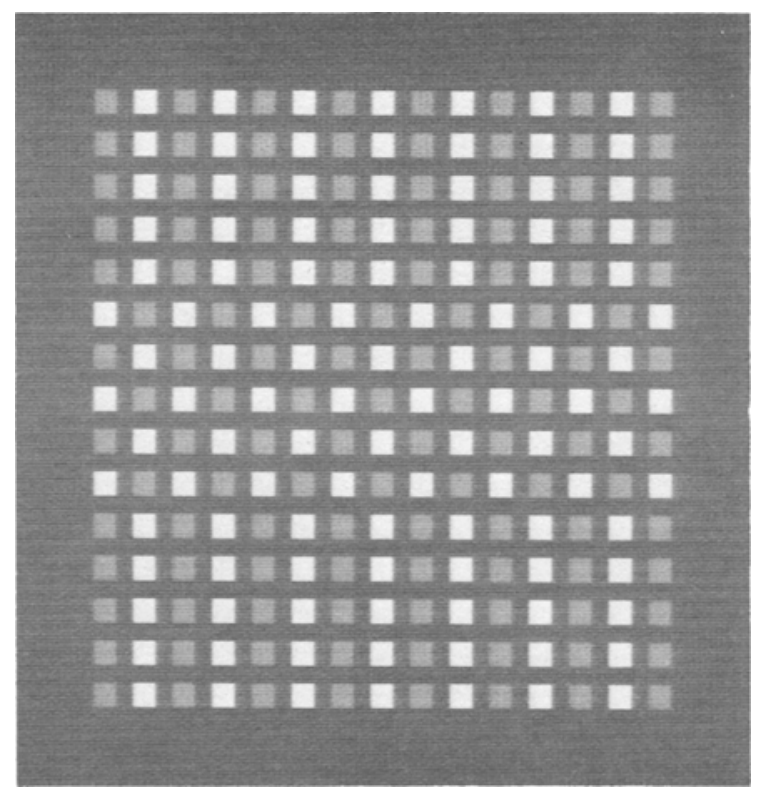

Figure 1. An example of the class of patterns investigated by Beck et al. (1983). The squares are of equal size and the contrast ratio between them is $4: 1$. The patterns and filter outputs pictured in this article are hardcopies of CRT images. Unfortunately, reproductions seldom accurately depict the dynamic range of the monitor, and contrast effects may therefore be diminished. taneously segregating it into two textures-stripes in the top and bottom regions and a checked region in the center. Beck et al. (1983) hypothesized that segregation occurs because squares of the same contrast are grouped into vertical stripes in the top and bottom regions but not in the center region. They proposed a model based on (1) feature detection and (2) the local linking of features to form texture elements through proximity, similarity, and good continuation. Julesz (1986) has also proposed that features link to form texture elements.

Beck et al. (1987) cast doubt on the explanation that texture segregation in patterns like those of Figures 1-3 are due to the lengths of the emergent vertical stripes in the pattern. The left panels of Figures 2 and 3 show patterns composed of squares whose areas differ by a ratio of 4:1. In Figure 2, the large and small squares are of equal contrast, and in Figure 3, the contrasts of the large and small squares differ by a ratio of $1: 4$. When they varied the contrast differences between the squares under two area-ratio conditions (equal-size squares as in Figure 1 and unequal-size squares as in Figure 2), Beck et al. (1987) found that area ratio and contrast ${ }^{1}$ are not independent attributes of texture segregation, but can cancel each other. For the patterns composed of equal-size squares, rated segregation increased as a negatively accelerated function of the contrast ratio of the two types of squares. For the patterns composed of unequal-size squares, rated segregation was a $U$-shaped function of the contrast ratio of the two types of squares, with a minimum at approximately the point where the contrast $\times$ area (areal contrast) of the elements was equal. In particular, a contrast ratio of 1:4, which produced good segregation when the squares were of equal size (Figure 1), produced poor segregation when the squares were of unequal size with an area ratio of 4:1 (Figure 3). This finding is puzzling from the point of view that texture segregation results from grouping the same-sized squares into vertical stripes in the top and bottom regions. Introducing a contrast difference as well as a size difference should facilitate such grouping and the segregation of the regions should improve. The tradeoff between contrast and area suggests that perceived texture segregation occurs strongly when the outputs of spatial-frequency channels are very different in the striped and checked regions of the texture pattern. This hypothesis does not involve explicit grouping processes that require differentiating texture elements from the background.

The left panel of Figure 2 shows a pattern having unequal-size squares and equal contrast. The middle panel shows the output of a simple spatial-frequency filter tuned to a vertical orientation and approximately to the fundamental spatial frequency of the textures, ${ }^{2}$ and the right panel shows the output of a higher spatial-frequency filter. (These filters are described in more detail below and in the Appendix.) Now consider the middle panel of Figure 2. When the excitatory region of a receptive field in that channel is centered over a column of large squares in the striped region, the receptive field is strongly stimu- 


\section{Element Type: Solid Squares Area Ratio 4 Contrast Ratio 1}
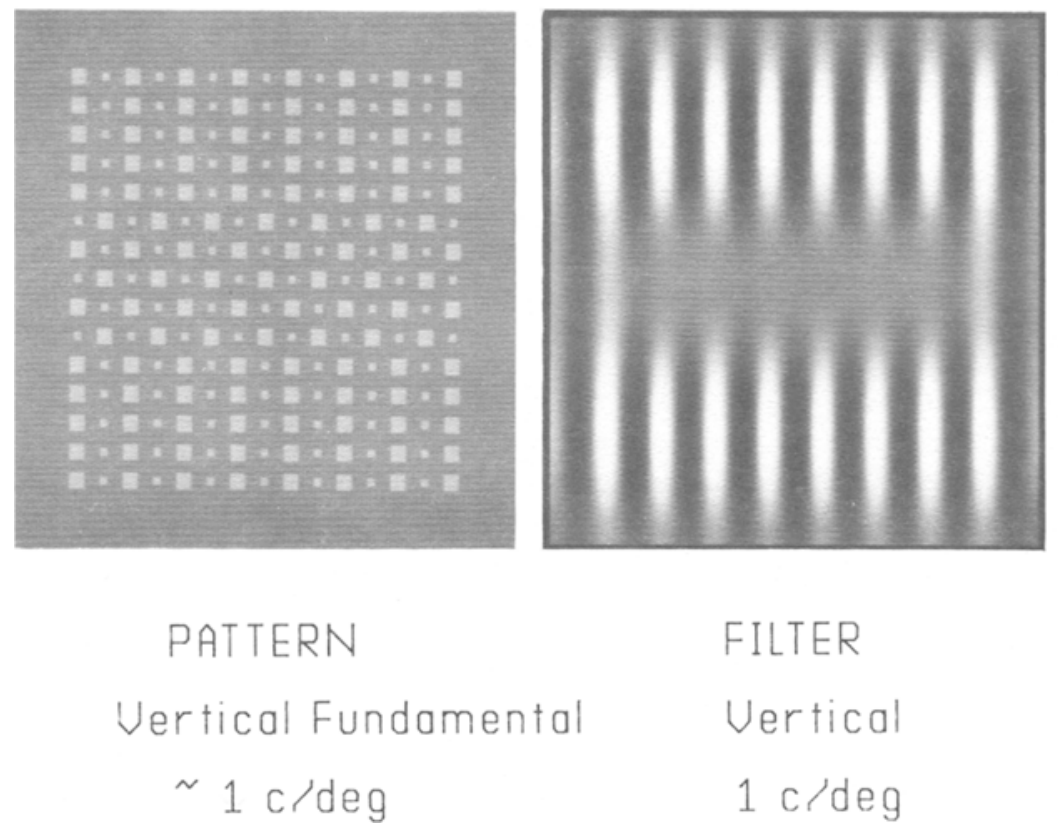

FILTER

Vertical

1 c/deg

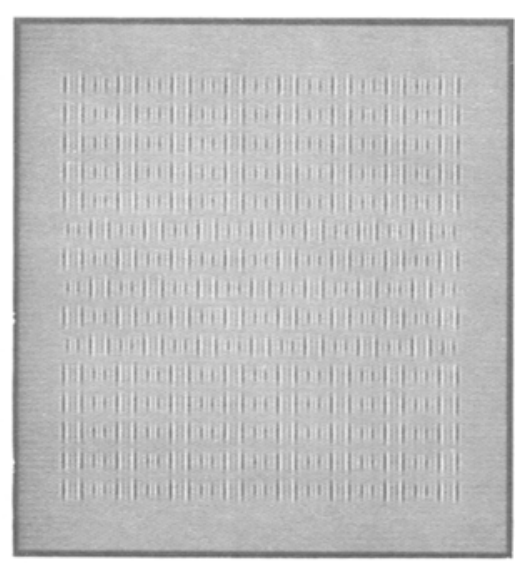

FILTER

\section{Vertical}

$11.3 \mathrm{c} / \mathrm{deg}$

Figure 2. The left panel shows a texture pattern in which the contrast of the squares is equal and the area ratio of the squares is $4: 1$. The center and right panels represent the responses to this pattern of filters tuned to vertical orientations, and 1 and 11.3 cpd, respectively.

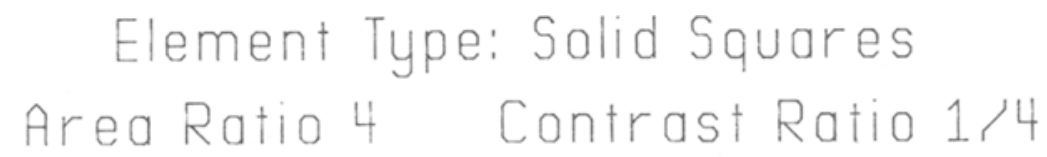

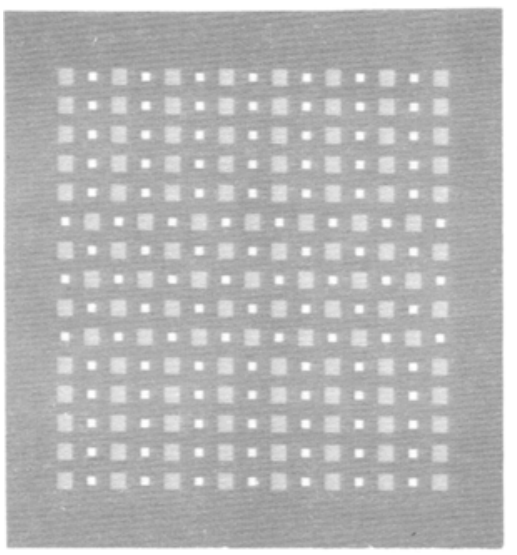
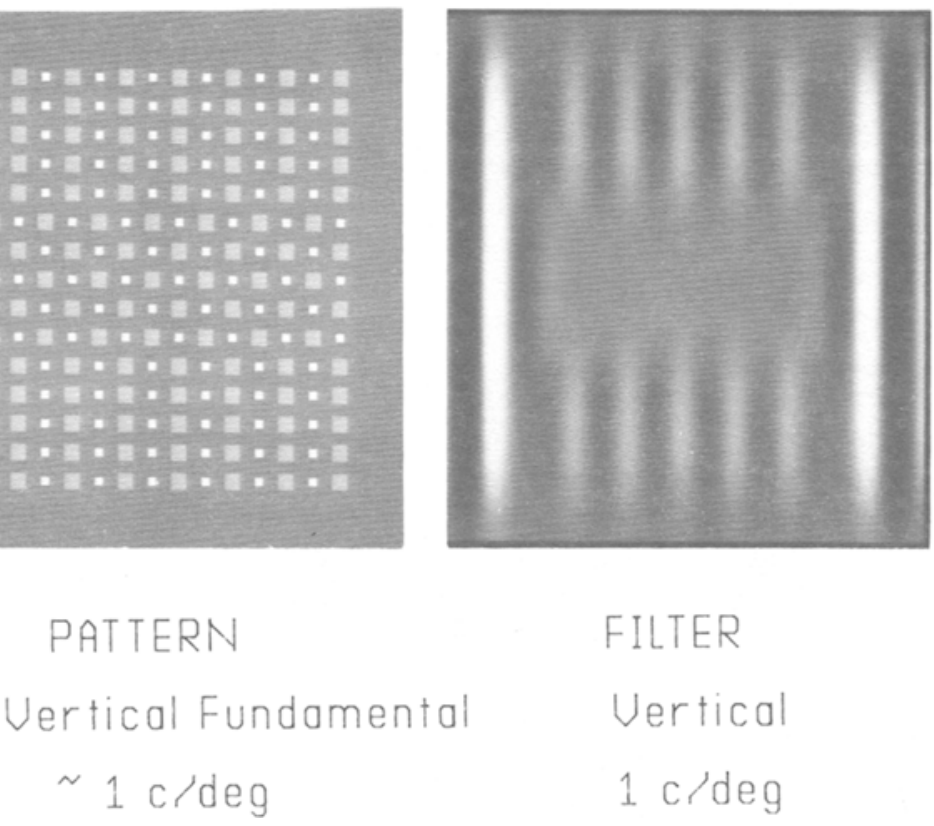

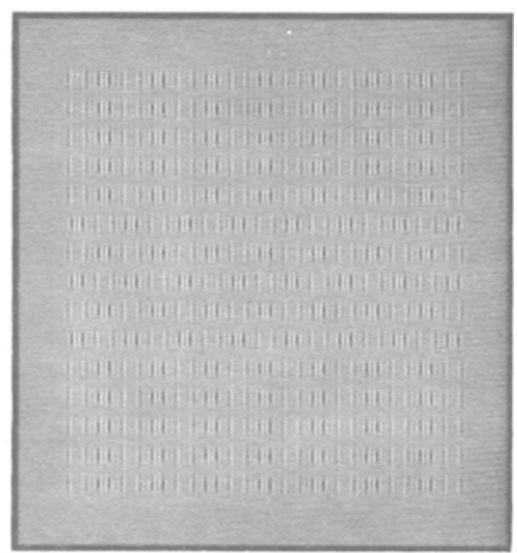

FILTER

Vertical

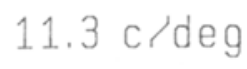

Figure 3. The left panel shows a texture pattern in which the contrast ratio of the squares is 1:4 and the area ratio of the squares is $4: 1$. The center and right panels represent the responses to this pattern of filters tuned to vertical orientations, and 1 and 11.3 cpd, respectively. 
lated by the large squares and weakly inhibited by the small squares. When the excitatory region is centered over a column of small squares, the receptive field is weakly stimulated by the small squares and strongly inhibited by the large squares. Thus, there is strong variation in activity with high outputs at the center of the large-square columns and low outputs at the center of the small-square columns. When the excitatory region of a receptive field is centered over either a large or a small square in the checked region of the pattern, the amounts of excitation and inhibition are approximately equal. Thus, the output at each spatial position is about the same, and there is less variation in activity (see middle region of output in the middle panel of Figure 2).

Figure 3 is like Figure 2 except that the contrast of the small squares in the pattern is now four times that of the large squares (so the areal contrasts are equal). Now when the excitatory region of a receptive field from the chan nel tuned to the fundamental frequency is centered over either a large or a small square in either the striped or the checked regions, the output is about the same, since the greater contrast has balanced out the smaller size of the squares.

\section{A SIMPLE SPATIAL-FREQUENCY CHANNELS MODEL}

Beck et al. (1987) presented evidence that when the output of the low spatial-frequency filters is similar in the top, middle, and bottom regions of patterns like those in Figure 1, texture segregation will not occur. The aim of this paper is to present a model embodying spatialfrequency channels and to compare its predictions to experimental data.

We conjectured that spontaneous strong texture segregation occurs only when there are differences in the mean or modulated activity of a channel or channels to the striped and checked regions of the texture pattern. We have attempted to construct a quantitative model that captures the kind of differences in the output of spatialfrequency filters that are visible in the middle panels of Figures 2 and 3, and that will predict an observer's ratings of perceived segregation. To do so requires considering the responses of all the filters. Figures 4 and 5 show the outputs of 39 filters to the patterns in Figures 2 and 3 , respectively. These outputs are displayed in a summary form in Figures 4 and 5 by taking a portion from the mid-

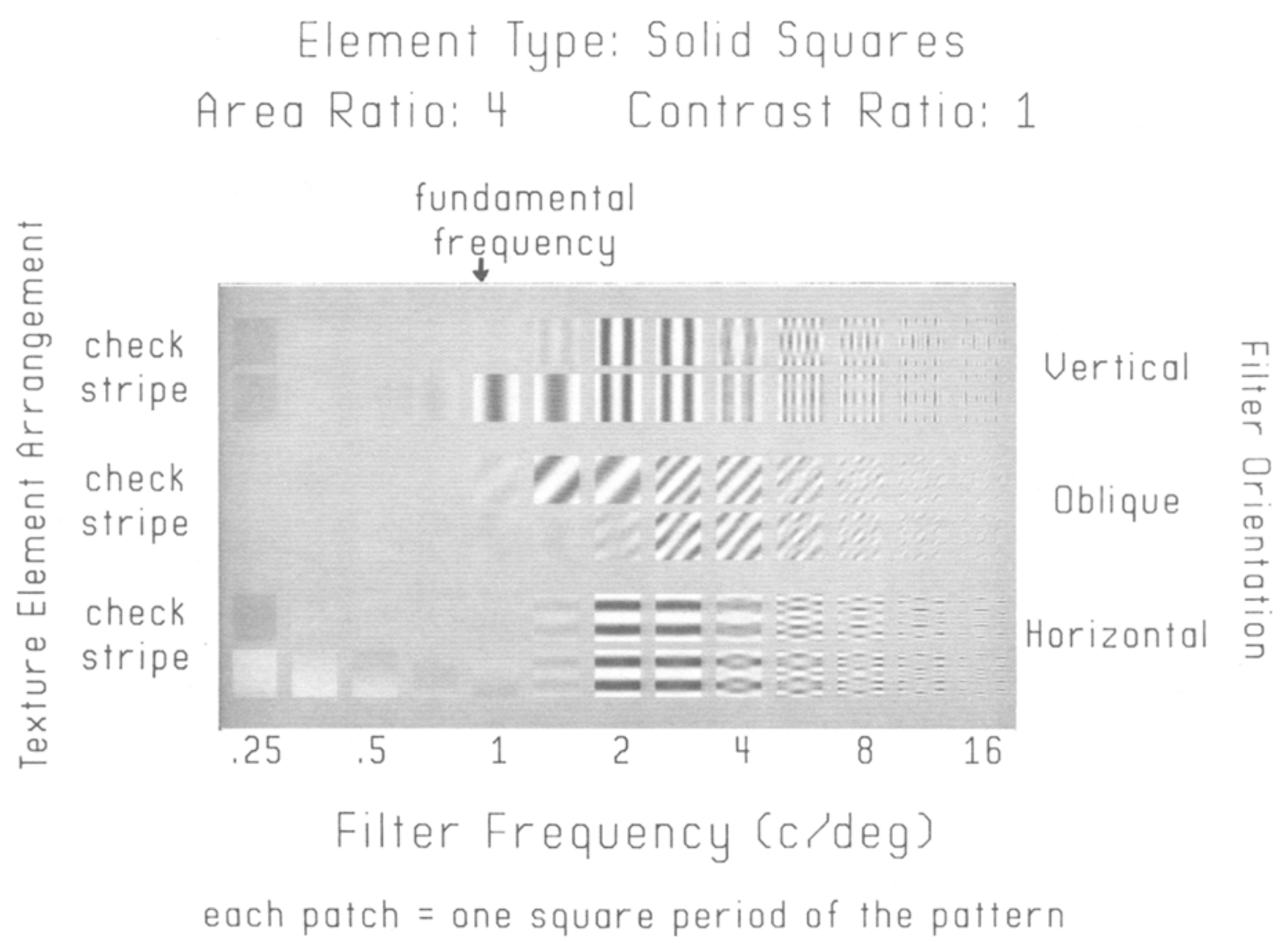

Figure 4. The responses of all 39 filters (13 spatial frequencies $\times 3$ orientations) to the pattern in the left panel of Figure 2 . Each patch in this display shows the response of a particular filter to one square period taken from either the checked or the striped region of the original pattern. The patches from the striped region were centered on a small square and the patches from the checked region were centered on a large square. The arrow shows the fundamental frequency for a vertically oriented filter. The fundamental frequency for an obliquely oriented filter is greater by $\sqrt{2}$. 

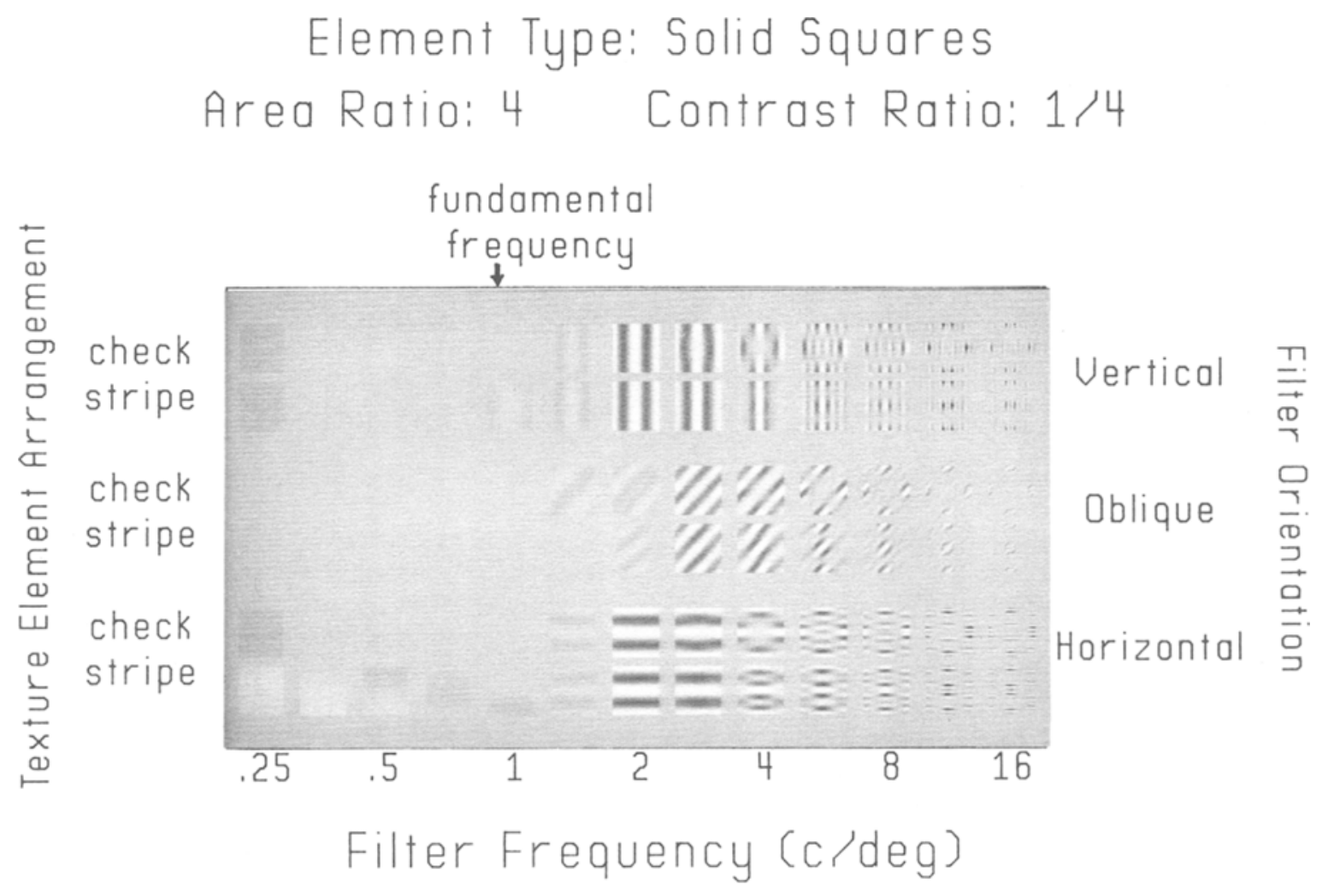

each patch = one square period of the pattern

Figure 5. The responses of all 39 filters (13 spatial frequencies $\times 3$ orientations) to the pattern in the left panel of Figure 3 . Each patch in this display shows the response of a particular filter to one square period taken from either the checked or the striped region of the original pattern. The patches from the striped region were centered on a small square and the patches from the checked region were centered on a large square. The arrow shows the fundamental frequency for a vertically oriented filter. The fundamental frequency for an obliquely oriented filter is greater by $\sqrt{2}$.

dles of the checked and striped regions of any one filter's output and displaying this pair of portions for each of many filters. Each portion equals one period of the pattern. The 39 filters come from all combinations of 13 different spatial frequencies (from left to right in 13 columns) and three different orientations-vertical, $45^{\circ}$ oblique, and horizontal (from top to bottom in three pairs of two rows each). Figures for textures composed of squares of equal size, analogous to Figures 4 and 5, can be found in Graham (1989).

It can be seen from Figure 4 that the channels that are particularly well suited to signal differences between the regions of the pattern are those that are (1) vertically oriented with a spatial-frequency sensitivity corresponding roughly to the fundamental vertical frequency of the striped region (1 and $1.44 \mathrm{cpd}$ ), and (2) diagonally oriented with a spatial-frequency sensitivity corresponding roughly to the fundamental oblique frequency of the checked region $(\sqrt{2}$ times the fundamental vertical frequency-1.44 and $2 \mathrm{cpd}$ ). When the large square is four times the area of the small square and the contrasts of the squares are equal, as in Figure 4, these channels show a large response to one region of the pattern but not to the other. When the areal contrast of the two squares is equal (i.e., when the small square is four times the contrast of the large square, as in Figure 5), these channels show no such difference in response.

For spatial frequencies lower than the fundamental frequency of the pattern, there is little response to either the checked or the striped regions by either the vertical or the oblique channels, because the receptive fields are so large that they average over adjacent rows and columns of squares. Channels tuned to spatial frequencies higher than the fundamental frequency respond to the edges of all the squares in the pattern. Although the pattern of activity is distributed differently in the striped and checked regions, the amounts of modulated activity in the striped and checked regions are similar.

\section{Characteristics of Channels}

Each channel is assumed to be a linear, translationinvariant filter. We modeled the receptive-field weighting functions with two-dimensional Gabor functions (as used, e.g., by Daugman, 1985; Watson, 1983). The 
weighting function in one direction is a Gaussian multiplied by a sinusoid, and in the perpendicular direction a Gaussian. The parameters of our model follow Watson's (1983) in that the half-amplitude, full bandwidth is one octave on the spatial-frequency dimension and $38^{\circ}$ on the orientation dimension. Our model is less complete than that of Watson in two respects: the fields have the same (even) symmetry, and we have not incorporated the decrease in acuity occurring with retinal eccentricity. In Figures 4 and 5, the variations in sensitivity with spatial frequency are not represented, but they are incorporated into the calculations of the model. Further details of the filters can be found in the Appendix.

\section{Combining Channel Outputs}

We now need to turn the channel outputs into a quantitative prediction of an observer's ratings of perceived texture segregation. Our first attempt is a crude measure of the degree to which there are gross differences between the outputs of one or more filters to the different texture regions. This simple model is an elaboration of Julesz's (1975) original statistical conjecture and is also in the spirit of recent attempts by a number of investigators (e.g., Caelli, 1982; Daugman, 1987; Turner, 1986) to use spatial-frequency filters to predict texture segregation. It differs from this earlier theoretical work in attempting to generate quantitative predictions of the strength of perceived texture segregation (rather than just distinguishing textures that would segregate from those that would not at all) so that these quantitative predictions can be compared to empirical results in a rigorous way.

1. Our simple model first computes the output of channels that are linear, translation-invariant filters tuned to many different spatial frequencies and orientations as described above. Let the output at position $(x, y)$ of the channel tuned to the $i^{\text {th }}$ frequency and the $j^{\text {th }}$ orientation be called $O_{i j}(x, y)$.

2 . The model then computes a spatially pooled response of each channel to the checked and to the striped region; in particular, the standard deviation of the outputs at different spatial positions within each region is computed. For example, the spatially pooled response of the $i j^{\text {th }}$ channel to the checked region is

$$
R_{i j}(\mathrm{ch})=\sum_{x} \sum_{\substack{\text { in } \\ \text { checked } \\ \text { region }}}^{N_{x}} \frac{\left[O_{i f}(x, y)-E\left(O_{i j}\right)\right]^{2}}{N_{x} \cdot N_{y}},
$$

where $N_{x}$ and $N_{y}$ are the numbers of spatial positions in the $x$ and $y$ directions in one period of the pattern and the summing is done over one period in the checked region. $E\left(O_{i j}\right)$ is the average value of $O_{i j}$ over these spatial positions. [Computations indicated, as expected, that $E\left(O_{i j}\right)$ to the striped and checked regions were highly similar. We therefore incorporated only differences in the stan- dard deviations of the outputs to the striped and checked regions in our quantitative model.] The same computation is carried out for the striped region, yielding $R_{i j}(\mathrm{st})$.

The model computes the difference between each filter's spatially pooled response to the checked and to the striped region, yielding a within-filter difference for the $i j^{\text {th }}$ filter of

$$
\operatorname{Diff}_{i j}=R_{i j}(\mathrm{ch})-R_{i j}(\mathrm{st}) .
$$

3. The model combines (pools) these within-filter differences across many spatial frequencies and orientations of filters, weighting the differences according to the observer's sensitivity to different orientations and spatial frequencies. In particular, the predicted value equals the following quantity:

$$
\sqrt{\sum_{i}^{N_{f}} \sum_{j}^{N_{A}}\left[\operatorname{Diff}_{i j} \cdot S_{\mathrm{obs}}\left(f_{i}, A_{j}\right)\right]^{2},}
$$

where $S_{\mathrm{obs}}\left(f_{i}, A_{j}\right)$ is the sensitivity of the observer to the $i^{\text {th }}$ frequency and $j^{\text {th }}$ orientation, $N_{f}$ is the number of frequencies (13-frequencies from .25 to $16 \mathrm{cpd}$ in steps of $\operatorname{rad} 2$ ), and $N_{A}$ is the number of orientations (three orientations: vertical, $45^{\circ}$ oblique, and horizontal).

4. Finally, the model assumes that the observer's ratings of perceived segregation are monotonically related to the predicted value shown above. Thus, the model does not predict that predicted values versus observer's ratings should fall on straight lines, but rather that they should fall on a monotonic function. Observed value (which can never go below 0 or above 4) will be an S-shaped function of predicted value.

We tried exponents of 1,3 , and 4 in the above equation (both for spatial pooling and for pooling across channels) as well as using the maximum, the minimum, and the maximum - the minimum of the within-channel difference. Conclusions using any of these alternate equations were identical to those presented here with an exponent of 2. It should be noted that our approach is very closely related to two approaches used by others in models of perceived texture segregation: (1) taking the Euclidean sum at each point of the output from two filters in quadrature phase (e.g., one having even-symmetric weighting functions and one having odd-symmetric weighting functions; Adelson \& Bergen, 1985; Bovik et al., 1987; Clark et al., 1987; Turner, 1986) and then comparing these sums in the two texture regions, or (2) using only even-symmetric weighting functions but pooling the rectified outputs over a small area around each point to produce a local energy measure (Bergen \& Adelson, 1988; Landy \& Bergen, 1988) and then comparing these local energy measures in the two regions. Either of these latter approaches leads naturally into a plausible model of how boundaries between the regions are actually found. This is a problem of great interest in its own 
right that has been discussed by a number of people (Caelli, 1985, 1988; Grossberg, 1987; Grossberg \& Mingolla, 1985), although it is not explicitly studied here.

\section{EXPERIMENTS}

In four experiments, we investigated the degree to which texture segregation is predicted by the simple model. In Experiment 1, we varied the background luminance and the areas of the squares composing the pattern. In Experiment 2, we compared line versus square texture elements. In Experiment 3, we investigated "duty cycle," or pattern density. In Experiment 4, we investigated how scaling of the texture patterns affects perceived segregation. In each experiment, the contrast ratios of the elements were varied. We define contrast ratio as the difference in luminance between the high-contrast elements and the background, divided by the difference between the low-contrast elements and the background.

\section{General Method}

\section{Stimuli and Apparatus}

The stimuli were generated by a Symbolics 3600 Lisp machine and were presented on a Tektronix 690 SR color monitor. Subjects viewed the stimuli from a distance of $6 \mathrm{ft}$. Consequently, one pixel subtended approximately $1.08 \mathrm{~min}$. The stimuli for all experiments consisted of variations of one basic pattern (see Figure 1). The pattern was composed of two types of texture elements (squares or lines), which could differ in size and/or contrast. The elements were arranged in 15 rows and 15 columns. In the top and bottom 5 rows, the elements were arranged to form alternating columns. In the central 5 rows, they were arranged in a checked pattern.

In the checked region, there were 37 of one type of element and 38 of the other. In the top and bottom (column) regions, there were 40 and 35 of the two types of elements, respectively. Except when noted, the horizontal and vertical center-to-center spacing of the elements in every pattern was 1.75 times the width of the larger elements in that pattern.

In all experiments except Experiment 1 (see below), the patterns were presented on a gray (16.1 fL) background, and the luminance of the larger texture elements was held constant at approximately $2 \mathrm{fL}$ above the background $(18.1 \mathrm{fL})$. The luminance of the other set of texture elements was assigned one of seven values ranging from 18.1 to $32.2 \mathrm{fL}$. The seven intensity values were chosen so that the intensity difference between the background and the second set of elements was $2^{n-1} \times 2.1 \mathrm{fL}$ for $n=1$ to 7 . This produced seven patterns having element-contrast ratios from 1 to 8 in steps of $\sqrt{2}$.

Experiment 1 consisted of two separate studies. In one study, the patterns were presented on a gray background as described above, except that a second set of patterns composed of squares that were darker than the background was included in addition to the patterns composed of squares that were lighter than the background. In the other study, the patterns were presented on a black background. The conditions for these studies will be described under Experiment 1 .

\section{Subjects}

For each experiment, 10 subjects were chosen from a pool of 33. Ten of the subjects participated in two or more of the experi- ments. Three of the subjects were graduate students in psychology, and the remaining 30 were undergraduates, psychology department staff, or people unaffiliated with the university. All but 3 of the subjects were naive about the purpose of the experiment. The 3 subjects who were not naive had minimal knowledge of the purpose of the experiment. All subjects had normal or corrected-tonormal vision, and all were paid for their participation.

\section{Procedure}

In each experiment, perceived segregation was evaluated using a 5-point category-rating scale ranging from 0 to 4 . The subjects were told that a rating of 0 meant that the three regions of the pattern were not distinguishable from each other without scrutiny. A rating of 4 meant that the three regions were very distinct and segregation was "immediate." The intermediate ratings signified intermediate perceptions of segregation from "barely perceptible" to "weak" to "moderate."

The subjects were seated in a dark room (ambient illumination $.05 \mathrm{ftc}) 6 \mathrm{ft}$ from the screen of the color monitor. A pattern representative of the stimuli in the experiment was presented on the screen. The experimenter described the structure of the pattern to the subject, pointing out that there were two types of texture elements and three different regions in the stimulus. The subjects were told that they would be asked to make a rating of the stimulus on the basis of how distinct the three regions were. Several of the experimental stimuli were then shown to the subjects to familiarize them with the range of variation of the patterns. The subjects were encouraged to base their judgments on their first impressions of the stimuli. They were instructed to keep their eyes on the center of the pattern, to treat each pattern as a whole, and not to scrutinize the patterns to find the boundaries between the three texture regions. They were also told not to base their judgments on how discriminable the two types of elements were from each other, but to judge only the distinctness of the three regions. The subjects were then given 10 practice trials, in which they told the experimenter the ratings of the stimuli.

Every subject participated in one experimental session. Each experimental session consisted of five blocks of trials. The number of trials per block varied between experiments, but in every experiment a block of trials consisted of one presentation of each of the stimulus patterns in that experiment, in random order. Thus, individual subject means were based on five ratings of each stimulus. At the beginning and end of every block of trials, the word "STOP' appeared in the center of the screen.

The subjects initiated each experimental trial by pushing a mouse button situated on a desk in front of them. An experimental trial consisted of the following sequence. A blue fixation " $X$ " was presented for $1 \mathrm{sec}$ in the center of a blank screen. Immediately after the offset of the fixation " $X$," the stimulus appeared and remained on the screen for $1 \mathrm{sec}$, after which it disappeared and the screen was blank. Throughout an experiment, including intervals during which the screen was blank, the background luminance of the screen remained constant at the value of the background for the stimuli in that experiment. After the stimulus disappeared from the screen, the subjects recorded their rating on a rating sheet and pushed the mouse button to initiate the next trial.

\section{Experiment 1 Squares-Area $\times$ Contrast}

Experiment 1 consisted of two separate studies designed to investigate more thoroughly how segregation of the texture patterns used by Beck et al. (1987) depends on the interaction of area ratio and contrast of the texture ele- 
ments. In each study, the effects of contrast differences between the two sets of texture elements were investigated using four different ratios of the areas of the two elements.

\section{Method}

Stimuli. In each of the patterns presented in this experiment, one set of squares measured 16 pixels $(17.28 \mathrm{~min})$ on a side. The other set of squares in the patterns measured $16,12,8$, or 4 pixels on a side $(17.28,12.96,8.64$, and $4.32 \mathrm{~min}$, respectively), creating four element-area ratio conditions $(1: 1,1.78: 1,4: 1$, and $16: 1)$. The center-to-center element spacing was 28 pixels (30.24 min), making the overall size of the pattern approximately $8^{\circ}$ in width and height.

In the first study of Experiment 1, the background was set at $16.1 \mathrm{fL}$. The two sets of squares in each stimulus pattern were both either lighter or darker than the background. As described in the
General Method section, seven element-contrast ratios were employed on either side of the background. When the squares were lighter than the background, the luminance of the larger texture elements was held constant at $18.1 \mathrm{fL}$ and the other texture elements were assigned one of seven values ranging from 18.1 to $32.2 \mathrm{fL}$. When the squares were darker than the background, the luminance of the larger elements was held constant at $14.1 \mathrm{fL}$ and the other texture elements were assigned one of seven values ranging from .03 to $14.1 \mathrm{fL}$. The 56 stimuli thus represented four element-area ratios $\times$ two sign of contrast $\times$ seven contrast ratios. In the second study, the background was set at $.05 \mathrm{fL}$. The 50 stimuli presented represented the partial combination of the four element-area ratios $\times 18$ contrast ratios. The constant-intensity $16-$ pixel square was set at $.26 \mathrm{fL}$. Twelve stimulus patterns were presented with a $1: 1$ element-area ratio. The variable-intensity $16-$ pixel square ranged from .26 to $9.48 \mathrm{fL}$. Fourteen stimulus patterns were presented with a $1.78: 1$ element-area ratio. The variable- (a)

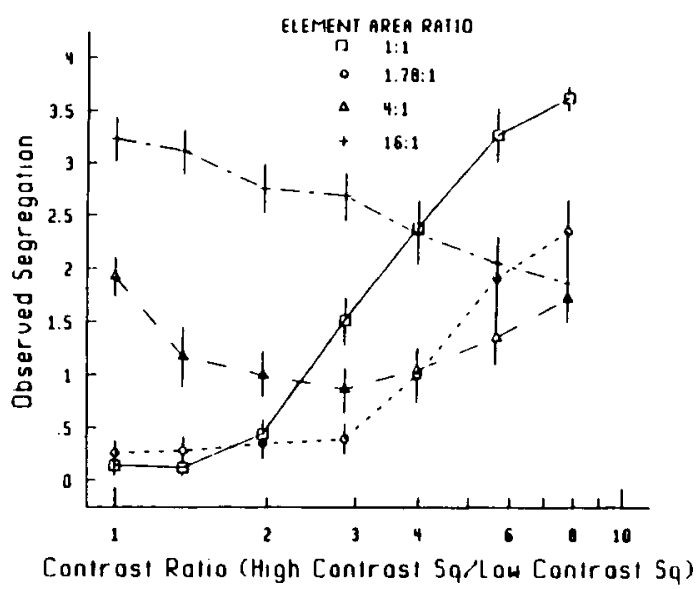

(b)

\section{Segregation}

(c)

\section{Black Background}

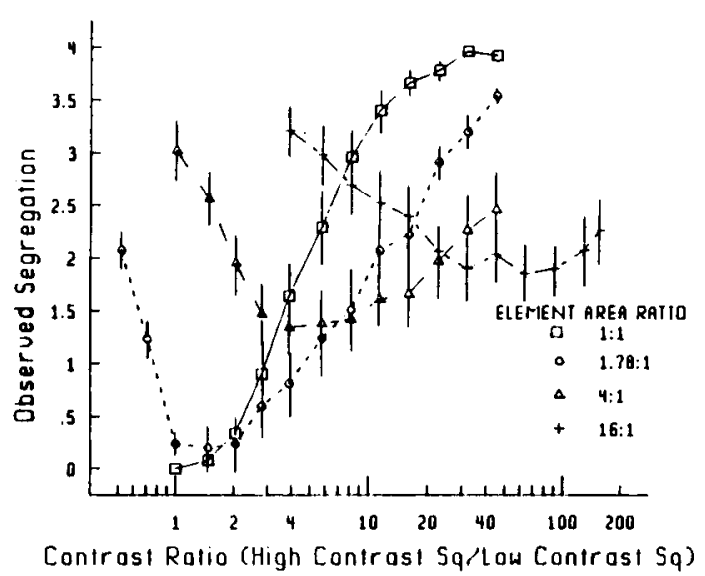

Figure 6. Mean observed segregation ratings in Experiment 1 as a function of the contrast ratio of the two types of squares for (a) patterns presented on a gray background in which the squares were above the background in luminance, (b) patterns presented on a gray background in which the squares were below the background in luminance, and (c) patterns presented on a black background. The vertical bars represent one standard error above and below the mean. 
intensity 12-pixel square ranged from .16 to $9.48 \mathrm{fL}$. Twelve stimulus patterns were presented with 4:1 and 16:1 element-area ratios, respectively. The variable-intensity 8 -pixel square ranged from .26 to $9.48 \mathrm{fL}$ and the variable-intensity 4-pixel square ranged from .87 to $32.2 \mathrm{fL}$. The range of intensities was covered in increments of approximately $.21 \mathrm{fL} \times \sqrt{2}$.

\section{Results and Discussion}

The results of Experiment 1 are shown in Figures 6a, $6 \mathrm{~b}$, and $6 \mathrm{c}$. Figure $6 \mathrm{a}$ shows the mean segregation ratings for the stimuli composed of squares that were above the gray background in luminance and Figure $6 \mathrm{~b}$ shows the mean ratings for the stimuli composed of squares that were below the gray background in luminance (hereafter, these two sets of stimuli will be referred to as the gray background-above and the gray background-below conditions). Figure $6 \mathrm{c}$ shows the mean segregation ratings for the stimuli with a black background. The mean segregation ratings are plotted against the contrast ratio of the two types of squares in each pattern. The vertical bars in the figures represent one standard error above and below the mean.

The interaction of element-area ratio and contrast. Figures $6 a-6 c$ show that perceived segregation is, in general, a U-shaped function with the minimum depending on the relative sizes of the squares, and shifting to larger contrast ratios as the element-area ratio increases.

Figure 7 presents the predicted segregation values from the simple linear spatial-frequency model. Only one set of predictions is shown because they are the same for the gray background-above, gray background-below, and black background conditions, except for a multiplicative constant. A comparison of Figure 7 with Figures 6a (gray background-above), $6 \mathrm{~b}$ (gray background-below), and $6 \mathrm{c}$ (black background) shows that there is good, but not per-
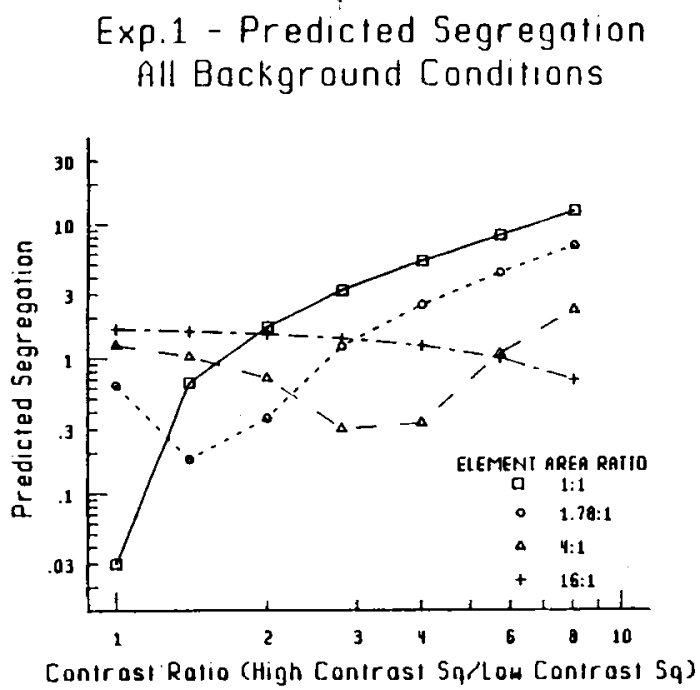

Figure 7. Predicted segregation values in Experiment 1 produced by the simple spatial-frequency channels model as a function of the contrast ratio of the two types of squares. The predictions are the same for all three background conditions (note that not all of the contrast ratios in the black background condition were modeled). fect, agreement between the observed and predicted values. For all element-area ratios, both the observed and the predicted ratings show minimum segregation around a point at which the areal contrast (area $\times$ contrast) of the two types of square is equal. For an element-area ratio of 1 , the minimum perceived segregation occurred when the contrast ratio was 1 . For an element-area ratio of 1.78:1, the minimum occurred at a constrast ratio between 1 and 2 . For $4: 1$, the minimum occurred at a contrast ratio between 2 and 4 , and for 16:1, the minimum occurred at a contrast ratio of approximately 20:1.

The effect of background. With a gray background, when the squares were below the background in luminance, perceived segregation was generally better than when the squares were above the background in luminance, particularly at high contrast ratios. The reason for this interaction is unclear. One possibility is that there may be an asymmetry in the response of the visual system to increments and decrements in intensity from the background (or adaptation) intensity. From what is known about adaptation, one might expect that as the contrast of the high-contrast squares increases (either above or below the background), light-adaptation processes would cause greater increases in contrast ratio to be needed to produce a change in perceived segregation. It is not clear whether light-adaptation processes occur at lower contrasts for stimuli with intensities above the adaptation level than for stimuli with intensities below the adaptation level, but it would explain the tendency for patterns with squares below the intensity of the background to yield greater segregation than patterns with the same contrast ratios whose squares were above the background in intensity.

The failure of the texture-segregation ratings to increase with increasing luminance of the small square on a black background, which can be seen in the 16:1 element-area ratio condition in Figure $6 \mathrm{c}$, also most probably reflects light-adaptation processes. The simple model's predicted value is linear with luminance. Experiments on light adaptation make it clear that the output from early sensory processes is linear throughout the range of luminances around the background luminance but compresses for luminances far above the background luminance (e.g., see review in Shapley \& Enroth-Cugell, 1985). The input to the channels should be the output from this nonlinearity, rather than the luminance of the squares.

A problem with the model. Although the largest effects in the experimental data are in accord with the simple model's predictions and point to the importance of differences in the outputs of simple, linear spatialfrequency channels to the different regions of the pattern, there is evidence that some other factor exerts a secondary, independent effect on segregation. This can be seen in Figures 8a-8c, which present the model's predictions plotted against the observed segregation ratings of our subjects. If the simple, linear spatial-frequency model were completely correct, the curves for the four element-area ratios would lie on top of one another in each panel. In other words, the observed segregation value should be 


\section{Exp.1 - Predicted us. Observed}

(a)

\section{Squores Above the Background}

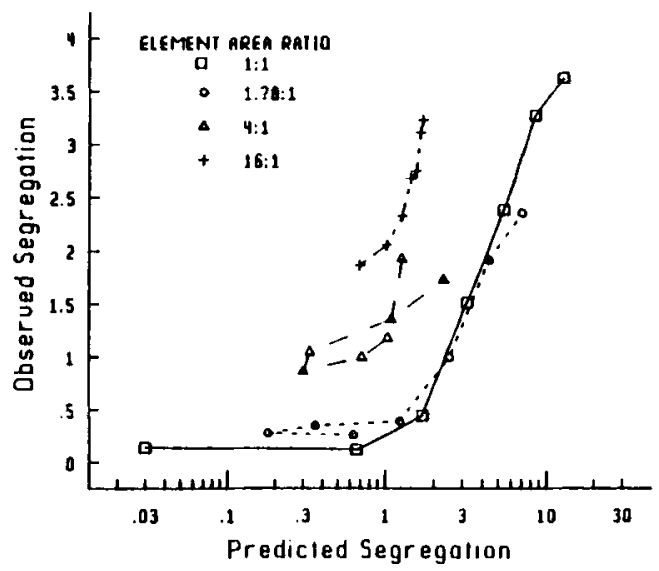

(b)

\section{Squares Below the Background}

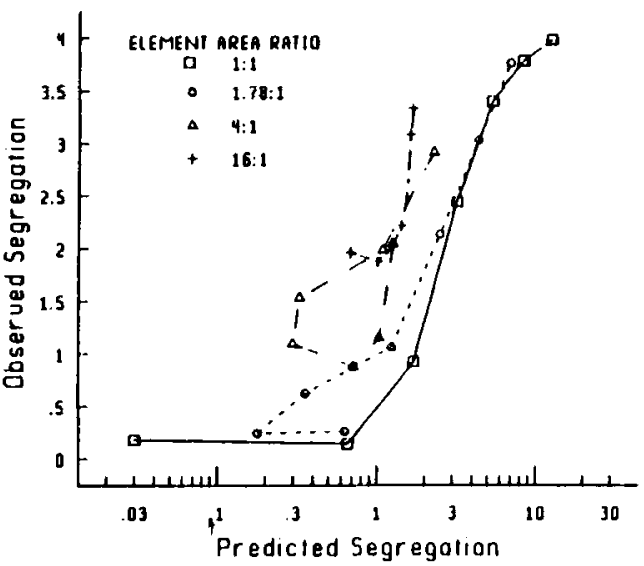

(c)

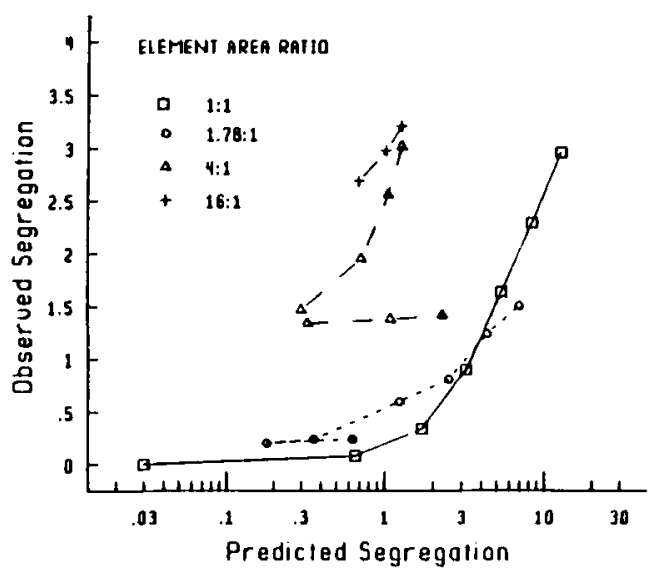

Figure 8. Mean observed segregation ratings in Experiment 1 plotted against predicted segregation values for (a) patterns presented on a gray background in which the squares were above the background in luminance, (b) patterns presented on a gray background in which the squares were below the background in luminance, and (c) patterns presented on a black background.

a monotonic transformation of the predicted value, and any given predicted segregation value should be associated with only one observed rating value. The fact that observed segregation ratings generally increase with element-area ratio as the two texture elements become more discrepant in size indicates that the model is insufficient to fully predict observed segregation. Some other factor influences perceived segregation, and this factor may involve information about the size differences between the elements, information that is carried by the channels sensitive to spatial frequencies higher than the fundamental frequency of the pattern. This possibility will be considered further in the General Discussion.

\section{Experiment 2}

\section{Lines-Area $\times$ Contrast}

For patterns composed of lines rather than squares, Beck et al. (1983) proposed that segregation is determined by the strength of linking of collinear line segments into long chains in the top and bottom regions of the pattern. In Experiment 2, we investigated whether perceived segregation in patterns composed of line elements could be explained by a spatial-frequency model without postulating linking processes. The effects of contrast differences between texture elements were investigated using two types of texture element (squares and lines), and four element-area ratios. 


\section{Method}

Stimuli. The 56 stimuli employed in this experiment represent a combination of two element shapes (squares and lines), four element-area ratios (which were different for squares and lines), and seven contrast ratios. When the elements were squares, the larger element was always 16 pixels (17.28 min) on a side; the second element in the pattern was a square, 16 pixels, 11 pixels (11.88 $\mathrm{min}$ ), 8 pixels (8.64 $\mathrm{min}$ ), or 4 pixels (4.32 $\mathrm{min}$ ) on a side. The resultant element-area ratios were $1: 1,2: 1,4: 1$, and 16:1, respectively. When the elements were lines, the larger element was always 16 pixels long and 2 pixels $(2.16 \mathrm{~min})$ wide; the second element was $16,11,8$, or 4 pixels long, and 2 pixels wide. The resultant area ratios were $1: 1,1.45: 1,2: 1$, and $4: 1$, respectively. The center-to-center element spacing was held constant at 28 pixels.

\section{Results and Discussion}

The results of Experiment 2 are shown in Figures 9a and $9 \mathrm{~b}$, for patterns composed of squares and lines, respectively.

(a)

\section{Exp.2 - Observed Segregation Squares}

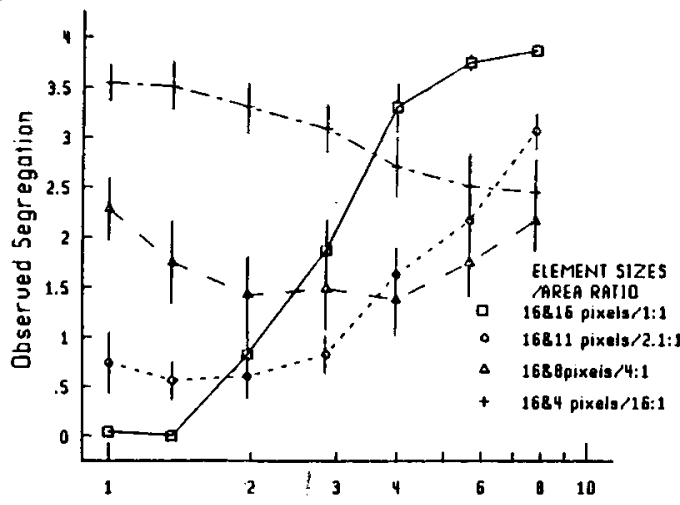

Contrast Rotio (High Conirast Sq/La Controst $\mathrm{Sq}$ )

(b)

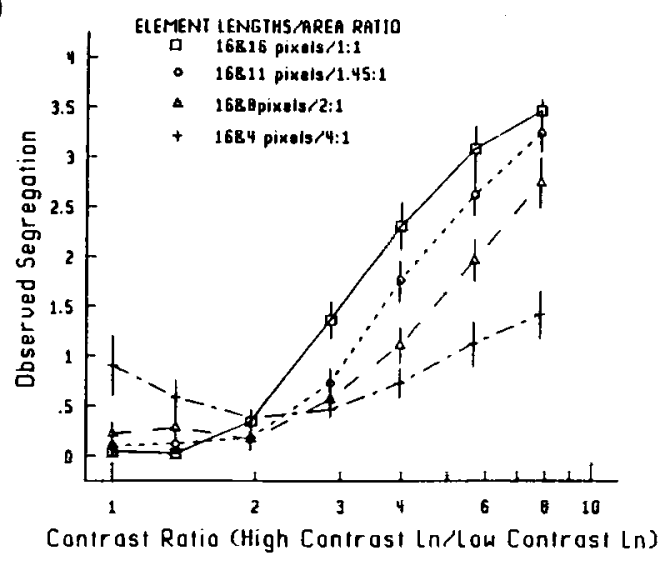

Figure 9. Mean observed segregation ratings in Experiment 2 as a function of the contrast ratio of the two types of elements for (a) patterns composed of squares and (b) patterns composed of lines. The vertical bars represent one standard error above and below the mean. (a)
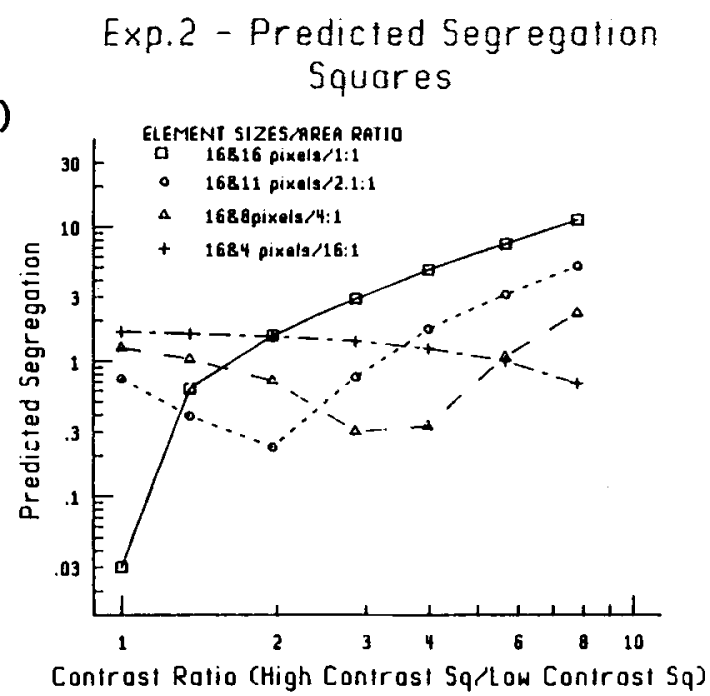

(b)

\section{Lines (2 pixels wide)}

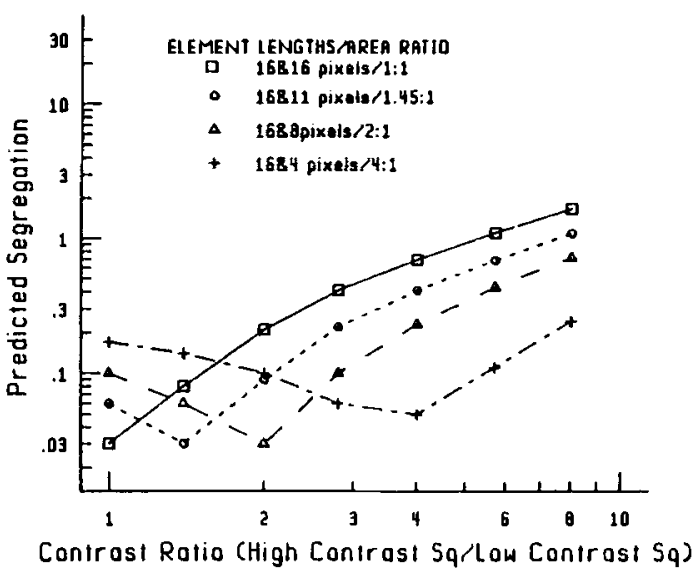

Figure 10. Predicted segregation values in Experiment 2 produced by the simple spatial-frequency channels model as a function of the contrast ratio of the two types of elements for (a) patterns composed of squares and (b) patterns composed of lines.

The interaction of element-area ratio and contrast. As in Experiment 1, perceived segregation depended on differences in areal contrast (contrast $x$ area) between the texture elements, with minimal segregation occurring at the point where the areal contrasts of the two types of elements were approximately the same, as predicted by the simple spatial-frequency model (Figures 10a and 10b). The interaction of element area and contrast supports the argument that the segregation of patterns composed of different arrangements of lines was not attributable to "emergent" features of the elements. If segregation of the line patterns had depended on the linking of the longer (16-pixel) lines into emergent, even longer lines, segregation should have been an increasing function of the contrast ratio between the two elements, regardless of their 


\section{Exp.2 - Predicled us. Observed Squares}

(a)

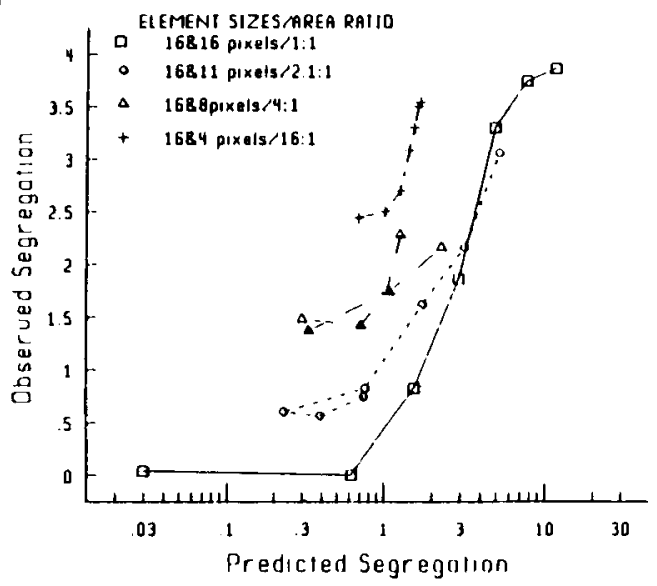

(b)

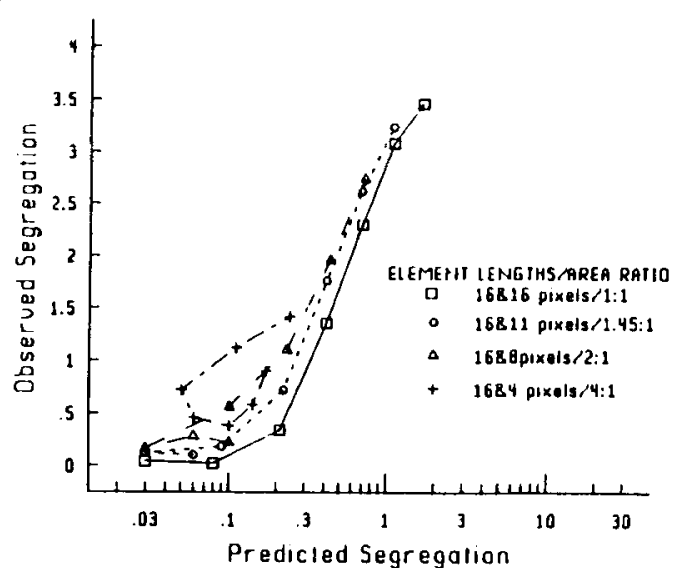

Figure 11. Mean observed segregation ratings in Experiment 2 piotted against predicted segregation values for (a) patterns composed of squares and (b) patterns composed of lines.

area ratio, since greater differences in contrast should have increased the linking of lines on the basis of similarity of contrast.

The effect of element shape. Patterns composed of squares tended to produce better segregation than did patterns composed of lines. This result was also found by Beck et al. (1983) and is predicted by the simple spatialfrequency model. This result could be caused by the fact that the lines occupied a smaller area than their square counterparts, and activation of spatial-frequency channels was thus "diluted" with the background.

Figures $11 \mathrm{a}$ and $11 \mathrm{~b}$ present the predicted segregation values plotted against observed segregation ratings. As in Experiment 1, the agreement between predicted and observed segregation is good but not perfect. There is much better agreement, however, between the observed and predicted segregation values for lines than for squares. For the textures composed of squares, the size differences between the squares affected perceived segregation. This can be seen in Figure 9a, which shows that the minimum segregation ratings increased with the size differences between the squares in the pattern. This result can not be explained by the simple model. Figure 10a shows that the perceived segregation of the patterns should be minimal and approximately equal when the areal contrasts have been equated. For the textures composed of lines, however, the size differences between the lines did not yield different minimum segregation ratings (Figure $9 b$ ). As shown in Figure 10b, this is in accord with the simple model. We will propose a possible reason for this difference in results in the General Discussion.

\section{Experiment 3 \\ Density (Duty Cycle)}

Beck et al. (1987) found that perceived segregation improved with increasing pattern density. Experiment 3 was conducted to determine whether the effects of pattern density (or duty cycle) on perceived segregation can be explained by the simple model. The center-to-center spacing of the elements was held constant at 14 pixels as the sizes of the squares in the patterns were varied, thus producing differences in the duty cycle of the patterns. The effects of contrast differences between two types of squares were investigated using two element-area ratios $(1: 1$ and $4: 1)$ and five sizes of large square $(12,10,8$, 6 , and 4 pixels).

\section{Method}

Stimuli. Seventy patterns were constructed through the combination of two element-area ratios (1:1 and $4: 1)$, five large-square sizes (12-, 10-, 8-, 6-, and 4-pixel squares), and seven contrast ratios. Thus, for the 1:1 element-area ratio condition, the patterns were composed of two sets of equal-size squares of $12,10,8,6$, or 4 pixels on a side $(12.96,10.80,8.64,6.48$, and $4.32 \mathrm{~min}$, respectively). In the $4: 1$ element-area ratio condition, the patterns were composed of 12- and 6-pixel squares, 10- and 5-pixel squares, 8- and 4-pixel squares, 6- and 3-pixel squares, or 4- and 2-pixel squares. The center-to-center element spacing was held constant at 14 pixels (15.12 min), thus creating patterns that were approximately $4^{\circ}$ in width and height.

\section{Results and Discussion}

The results of Experiment 3 are shown in Figures 12a and $12 \mathrm{~b}$. Figure $12 \mathrm{a}$ shows the mean segregation ratings for the 1:1 element-area ratio condition, and Figure 12b shows the mean ratings for the $4: 1$ element-area ratio condition. As in Experiments 1 and 2, perceived segregation was minimal at or around the point at which the areal contrasts (area $\times$ contrast) of the two types of texture elements were equal.

As large-square size (and therefore pattern density and duty cycle) decreased, there was a corresponding decrease in perceived segregation for both element-area ratios. The same trend is visible in Figures 13a and 13b, the predicted segregation values from the simple model. As element size 
was reduced, the density of texture elements decreased and the amount of empty background space increased, thus diluting the effects of contrast differences between the squares, and reducing the activity of the channels. This reduction in the activity of the channels would have the effect of reducing differences in the modulated activity of the channels to the different regions of the pattern, causing a corresponding decrease in perceived segregation. (This is similar to the result obtained in Experiment 2. The patterns composed of squares, which had a higher density than the patterns composed of lines, yielded stronger segregation.) Remembering that the observed rating is only expected to be some monotonic transformation of the predicted values, it can be seen that the predicted

(a)

\section{Exp.3 - Observed Segregation 1:1 Areo Ratio}

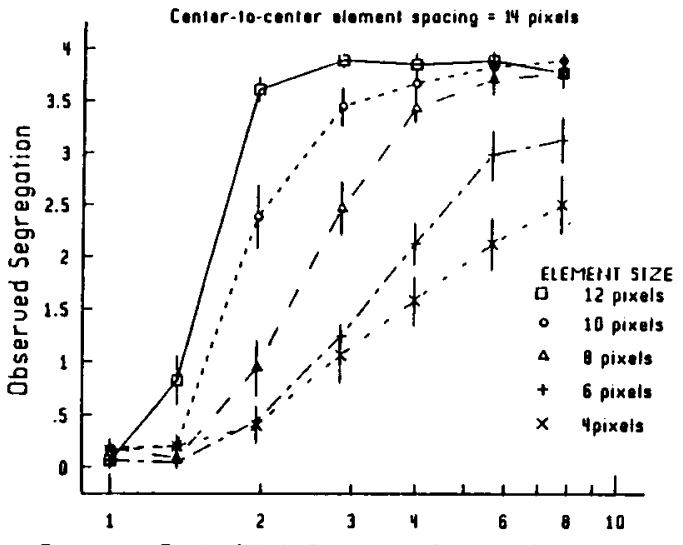

Contrast Ratio (High Conirast SqRow Contrast Sq)

(b)

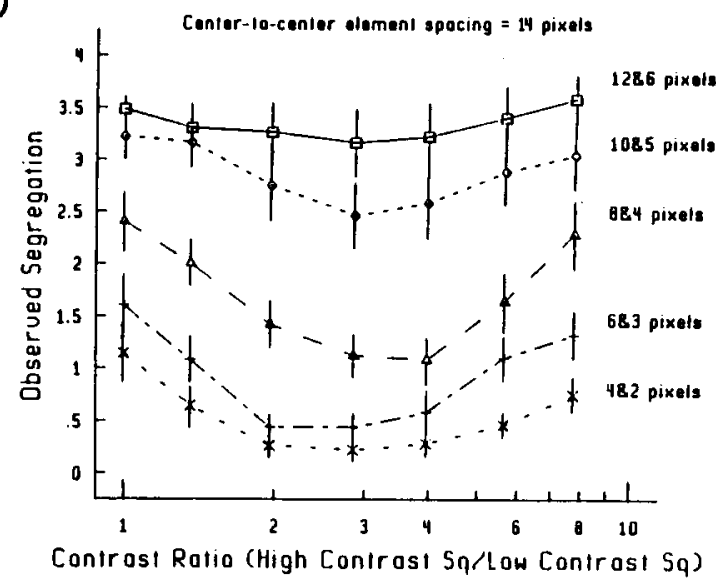

Figure 12. Mean observed segregation ratings in Experiment 3 as a function of the contrast ratio of the two types of squares for (a) patterns composed of squares with an area ratio of $1: 1$ and (b) patterns composed of squares with an area ratio of 4:1. The vertical bars represent one standard error above and below the mean. (a)

$$
\begin{gathered}
\text { Exp.3 - Predicled Segregation } \\
\text { 1:1 Area Rotio }
\end{gathered}
$$

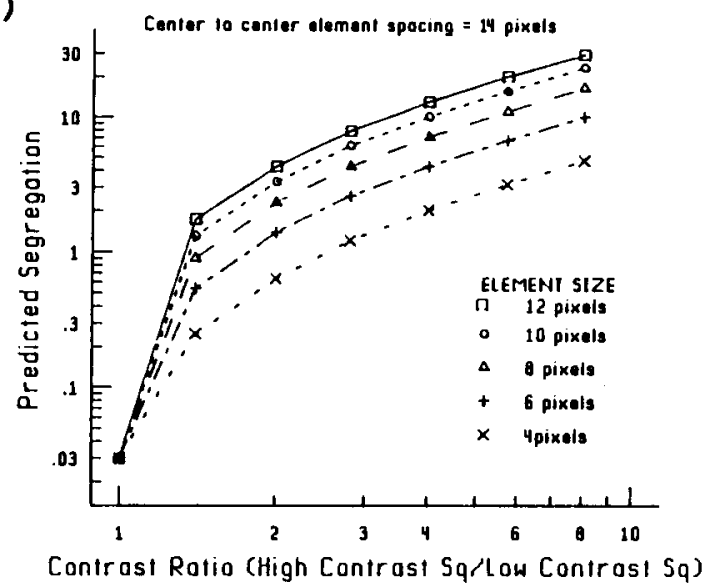

(b)

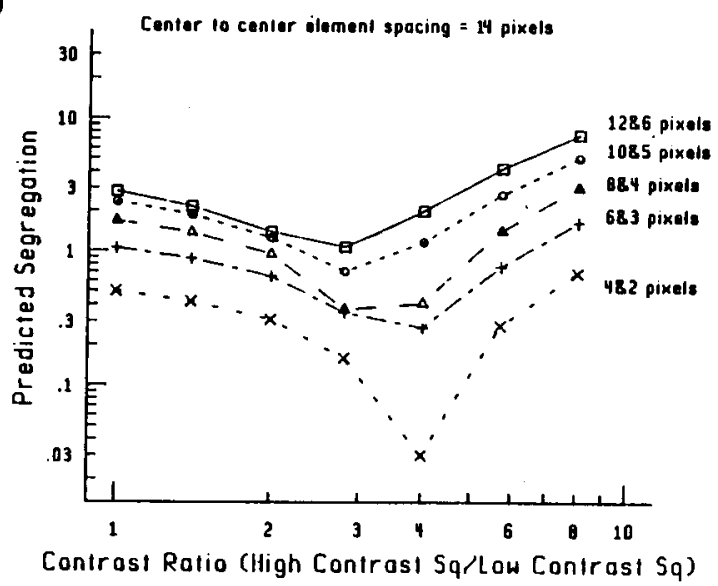

Figure 13. Predicted segregation values in Experiment 3 produced by the simple spatial-frequency channels model as a function of the contrast ratio of the two types of squares for (a) patterns composed of squares with an area ratio of 1:1 and (b) patterns composed of squares with an area ratio of 4:1.

segregation curves agree quite well with the observed segregation curves, as is clear in Figures 14a and 14b.

As in Experiment 1, however, the outputs of the simple, linear spatial-frequency model do not fully predict observed segregation (see Figures 14a and 14b). If the simple, linear spatial-frequency model were completely correct, any given predicted segregation value would be associated with only one, or a close range of, observed segregation values. For the 1:1 element-area ratio condition (Figure 14a), the model does a very good job of predicting observed segregation with decreasing density of the pattern elements. The curves lie approximately on top of one another. The superposition is not as good in the $4: 1$ element-area ratio condition (Figure 14b). In ad- 


\section{Exp. 3 - Predicled us, Observed 1:1 Area Rolio}

(a)

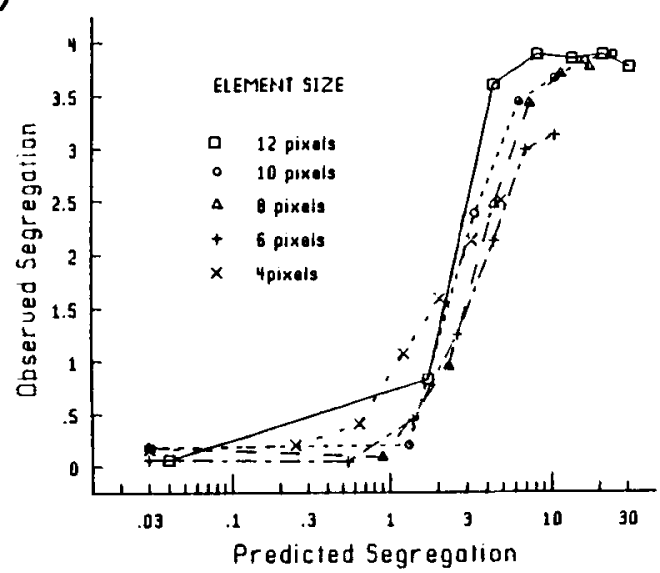

(b)

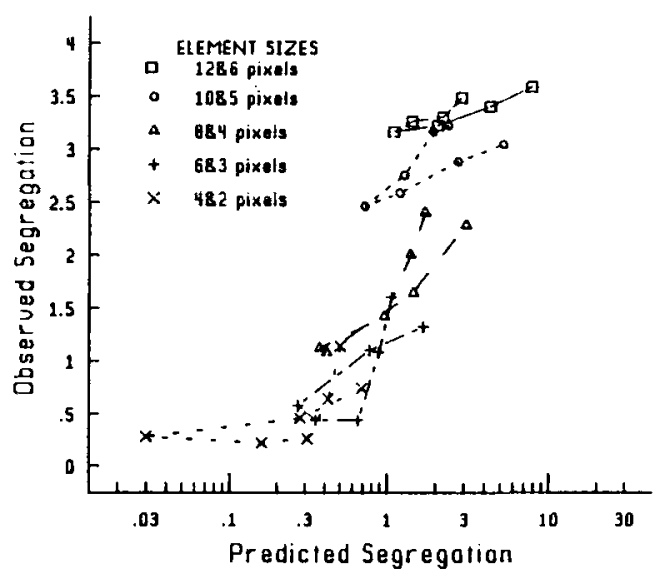

Figure 14. Mean observed segregation ratings in Experiment 3 plotted against predicted segregation values for (a) patterns composed of squares with an area ratio of $1: 1$ and (b) patterns composed of squares with an area ratio of $4: 1$.

dition, a comparison of Figures 14a and 14b shows the same predictive failure that occurred in Experiment 1. For most predicted segregation values, the observed ratings in the 4:1 element-area ratio condition are considerably higher than the corresponding ratings in the 1:1 elementarea ratio condition.

\section{Experiment 4 Pattern Scaling}

In Experiment 4, we examined a property of our patterns that is not generally shared by patterns usually used to investigate grouping processes. Wertheimer (1923) observed that the grouping of a set of elements does not change with viewing distance or the magnification of the pattern. Green, Wolf, and White (1959) found that, as long as the relative element sizes and separations remain constant, the absolute size of the pattern does not affect texture discrimination. In contrast, Beck et al. (1983) found that patterns like Figure 1 fail to scale. When the sizes and separations of the squares were reduced by one half, perceived segregation increased. This indicates that segregation depends on the absolute sizes of the elements and their separations.

This observation is consistent with the view that perceived segregation is mediated by the outputs of spatialfrequency channels. Contrast sensitivity is generally highest at spatial frequencies ranging from 2-8 cpd, depending on experimental conditions (Graham, in press). The patterns presented in Experiments 1 and 2 had a period of 56 pixels (the distance between the centers of two columns of the same type of square), which translates to approximately $1 \mathrm{cpd}$. Figures 4 and 5 show that the spatial-frequency channel that gives the best information for segregation is one that matches the period of the pattern. For the patterns presented in Experiments 1 and 2 , this channel would have peak output at a spatial frequency of around $1 \mathrm{cpd}$. By either increasing the viewing distance or proportionately decreasing the sizes of the elements and their separations, the period of the pattern can be decreased, thus increasing the spatial frequency that carries the most information about differences between the striped and checked regions of the pattern. Reducing the period of the pattern should increase perceived segregation, up to the point where the fundamental frequency component of the pattern has a spatial frequency at the peak of the contrast-sensitivity function. Further reduction of the period of the pattern should lead to a decrease in perceived segregation because the fundamental frequency component of the pattern will be of a spatial frequency that is higher than that at the peak, thus entering a range where contrast sensitivity decreases.

In Experiment 4, we tested this prediction of the spatialfrequency model. The period of the pattern was reduced by decreasing the sizes and separation of the squares making up the pattern. The effects of contrast differences between the two types of squares were investigated under three element-area ratios $(1: 1,4: 1$, and 16:1) and four fundamental frequency conditions $(1,2,4$, and $8 \mathrm{cpd})$.

\section{Method}

Stimuli. Seventy-seven stimuli were constructed through the partial combination of three element-area ratios $(1: 1,4: 1,16: 1)$, four fundamental frequencies $(1,2,4$, and $8 \mathrm{cpd})$, and seven contrast ratios. The three element-area ratio conditions were equal-size squares (1:1 element-area ratio), unequal-size squares with a ratio of areas of $4: 1$, and unequal-size squares with a ratio of areas of 16:1. The four fundamental frequencies were $1,2,4$, and $8 \mathrm{cpd}$, which corresponded to center-to-center element separations (onehalf periods) of 28 pixels ( $30.24 \mathrm{~min}), 14$ pixels $(15.12 \mathrm{~min}), 7$ pixels (7.56 $\mathrm{min}$ ), and 4 pixels (4.32 $\mathrm{min}$ ), respectively. The centerto-center element separation was held constant at 1.75 times the width of the larger square in the pattern. ${ }^{3}$ The four large-square sizes were $16,8,4$, and 2 pixels on a side $(17.28,8.64,4.32$, and $2.16 \mathrm{~min}$, respectively). The effect of reducing the element size 


\section{Exp. 4 - Observed Segregation}

(a)

1:1 Area Ratio

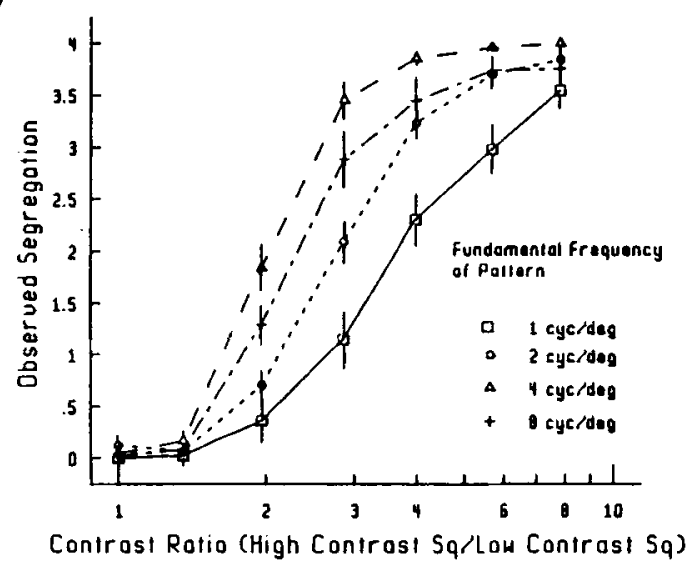

(b)

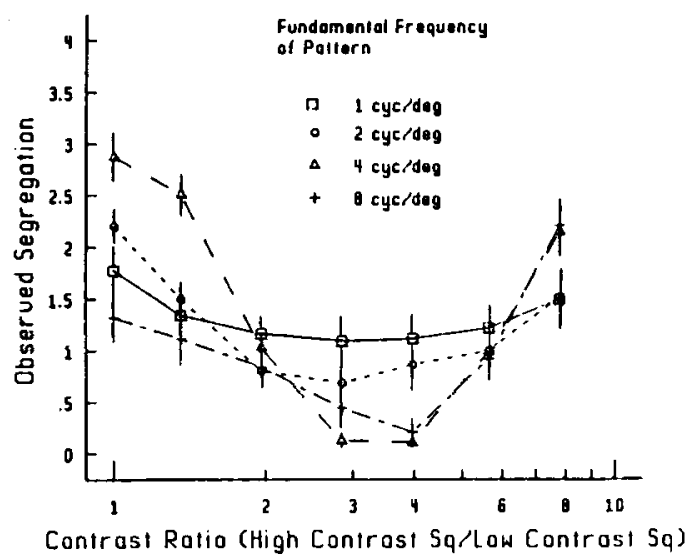

(c)

16:1 Areo Ratio

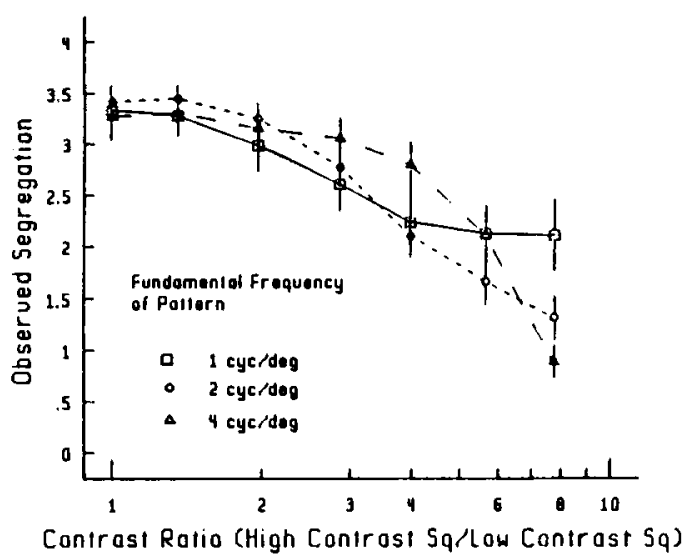

Figure 15. Mean observed segregation ratings in Experiment 4 as a function of the contrast ratio of the two types of squares for (a) patterns composed of squares with an area ratio of 1:1, (b) patterns composed of squares with an area ratio of 4:1, and (c) patterns composed of squares with an area ratio of 16:1. The vertical bars represent one standard error above and below the mean.

and separation was to decrease the size of the whole pattern, as well as its period. The patterns with a fundamental frequency of 1 cpd measured $7.56^{\circ}$ in height and width. The patterns with fundamental frequencies of 2,4 , and 8 cpd measured $3.78^{\circ}, 1.89^{\circ}$, and $1.08^{\circ}$, respectively, in height and width.

\section{Results and Discussion}

The results of Experiment 4 are presented in Figures $15 a-15 c$ for the $1: 1,4: 1$, and $16: 1$ element-area ratios, respectively.

The interaction of element-area ratio and contrast. As in the previous experiments, perceived segregation is a U-shaped function of contrast ratio, with a minimum around the point at which the area $\times$ contrast of the two texture elements is equal. Figures $16 a-16 c$ show the predicted segregation values produced by the simple spatial-frequency model. A comparison of the predicted and observed segregation curves shows that the general shapes of the observed segregation curves for each element-area ratio are fairly well predicted by the model, as are the contrast ratios at the points of minimum segregation for the three element-area ratios.

The effect of fundamental frequency. Figures $15 \mathrm{a}-15 \mathrm{c}$ show that perceived segregation of our patterns varied with their period. Some aspects of this variation in perceived segregation are predicted by the simple spatial-frequency model (Figures 16a-16c). According to the model, the pattern that should be most easily segregated, at a given contrast ratio, is that whose fundamental spatial frequency is approximately $4 \mathrm{cpd}$. Patterns with higher or lower fundamental frequencies should be more difficult to segregate. This prediction is consis- 


\section{Exp.4 - Predicted Segregation}

(a)

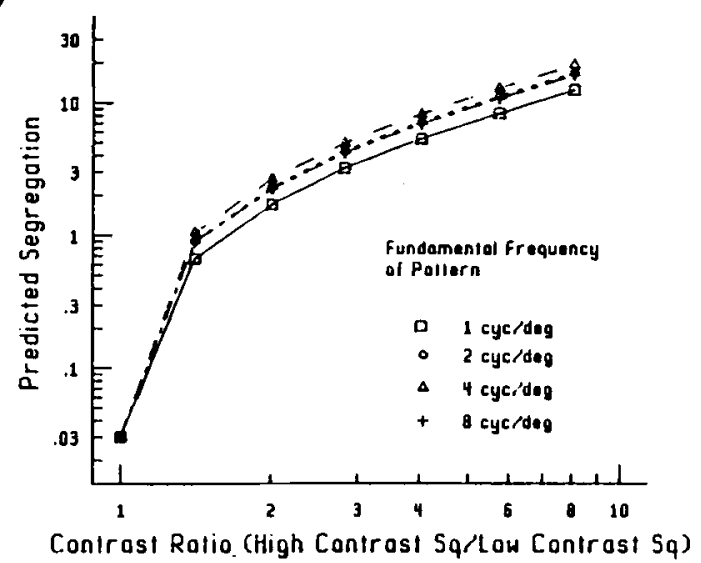

(b)

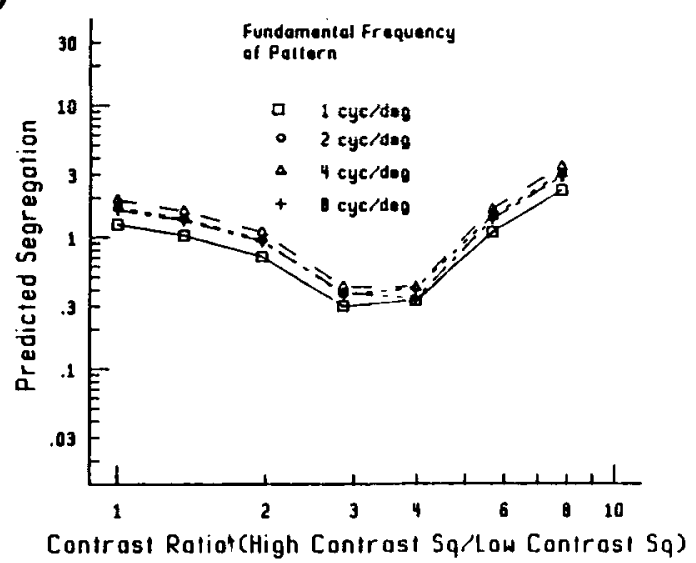

(c)

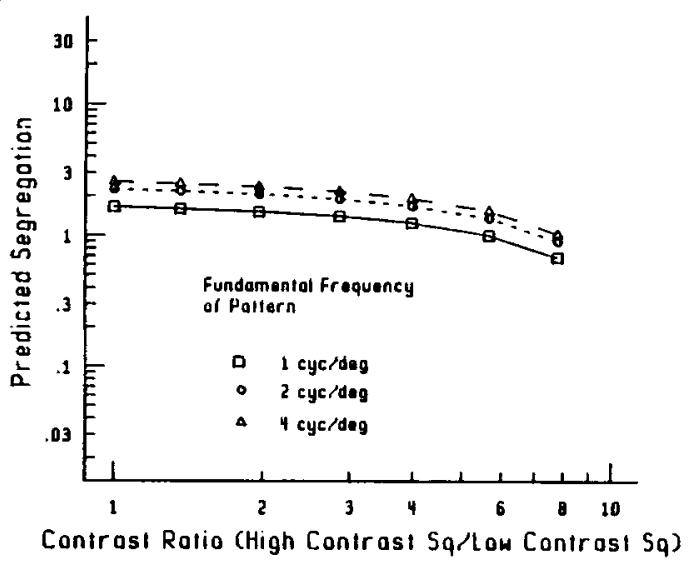

Figure 16. Predicted segregation values in Experiment 4 produced by the simple spatial-frequency channels model as a function of the contrast ratio of the two types of squares for (a) patterns composed of squares with an area ratio of 1:1, (b) patterns composed of squares with an area ratio of 4:1, and (c) patterns composed of squares with an area ratio of 16:1.

tent with many of the observed segregation ratings, with a few striking exceptions in the 4:1 and 16:1 element-area ratio conditions. The contrast ratio at which minimum observed segregation occurs in these conditions is well predicted by the model, but note that the observed segregation curves cross over dramatically, whereas the predicted curves just move up or down, for the most part, depending on fundamental frequency. For very low contrast ratios, the ordering of curves is roughly from the most visible on top to the least visible fundamental frequencies (as was built into the model by weighting the predictions by a particular sensitivity function). ${ }^{4}$ In the trough, however, ordering is different; patterns having low fundamental frequencies still segregate quite well (contradicting the model), whereas patterns with high fun- damental frequencies do not segregate at all (agreeing with the model). The failure of the model to predict this crossover can also be seen in Figures $17 \mathrm{~b}$ and $17 \mathrm{c}$, where there is a wide range of observed segregation values associated with any particular predicted value. The model predicts that there should be only one observed value for each predicted value because the observed values are hypothesized to be only a monotonic transformation of the predicted values. Figure 17a shows that for the 1:1 element-area ratio, there is good agreement between the predicted and observed segregation values. The effects of changing the fundamental frequency discussed above were also obtained by Sutter (1987) with patterns of constant size. Instead of reducing the overall size of the patterns, Sutter maintained the overall size of the patterns 


$$
\text { Exp. } 4 \text { - Predicted us. Observed }
$$

(a)

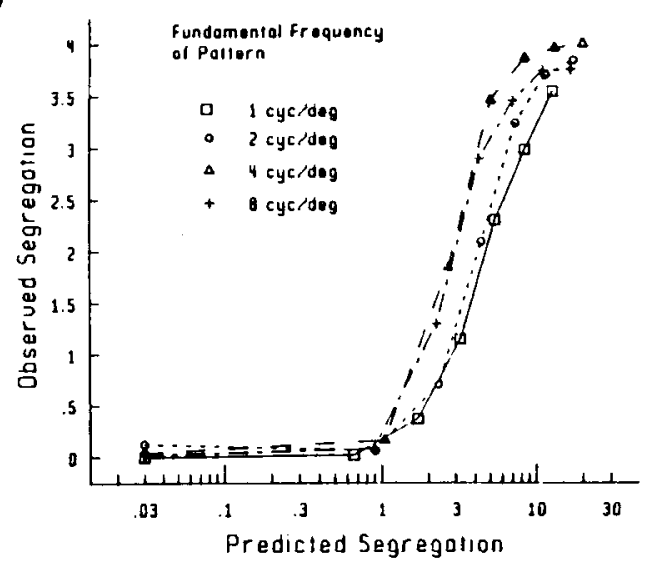

(b)

4:1 Areo Rolio

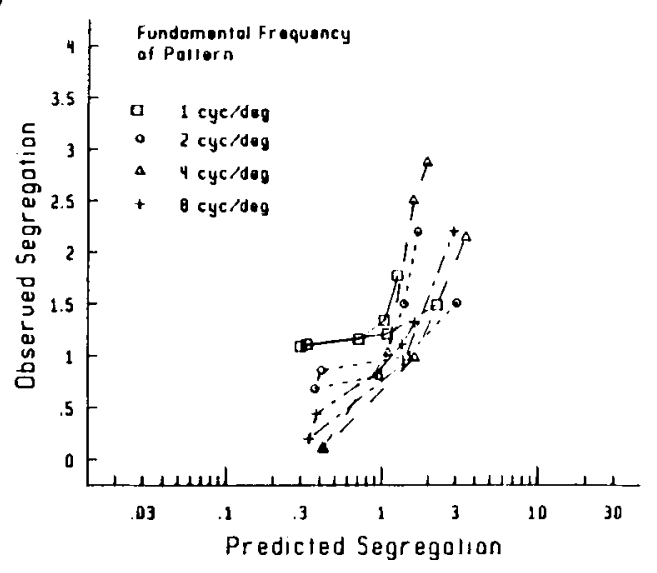

(c)

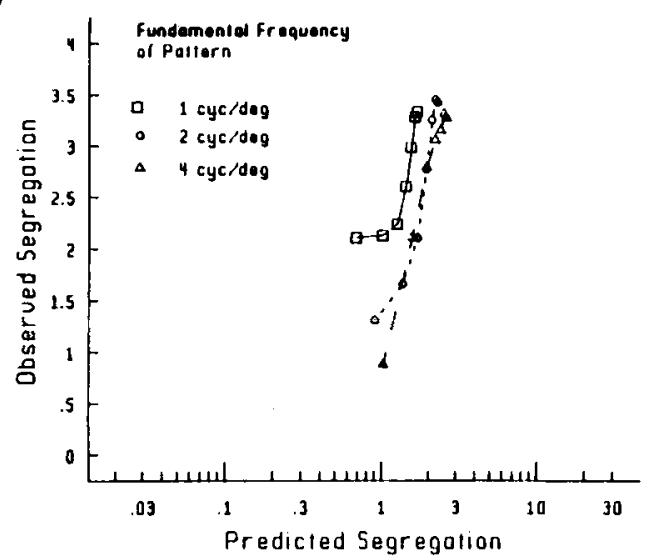

Figure 17. Mean observed segregation ratings in Experiment 4 plotted against predicted segregation values for (a) patterns composed of squares with an area ratio of $1: 1$, (b) patterns composed of squares with an area ratio of $4: 1$, and (c) patterns composed of squares with an area ratio of 16:1.

by increasing the number of rows and columns of elements.

\section{GENERAL DISCUSSION}

Two principal results of the experiments reported here are predictable from our simple model: texture segregation tended to be a $\mathrm{U}$-shaped function of the contrast ratio, and it was minimal when the area $\times$ contrast of the large and small elements making up a pattern were approximately equated.

Two aspects of the results are, however, quite different from those predicted from a simple linear pooling of within-filter differences weighted by the contrastsensitivity function (Equation 3). First, the minimum segregation rating was a function of the size difference between the large and small squares making up a pattern. Figures $6 a-6 c$ and 9a show that in Experiments 1 and 2, the minimum segregation ratings increased as the size differences, or equivalently the area ratios, between the large and small squares in a pattern became larger. If areal contrast were the only factor affecting segregation, the segregation ratings should have been the same when the area $\times$ contrast between the large and small squares composing a pattern were equated. The predicted minima for different area ratios in Figures 7 and 10a are approximately equal (except for the 1:1 element-area ratio condition). Size differences between the large and small squares also affected the observers' ratings in Experiment 4 , in which ratings at equal areal contrasts (a 1:4 
contrast ratio and 4:1 element-area ratio) varied with the scaling of the pattern. Figure $15 \mathrm{~b}$ shows that the dip in the U-shaped function becomes less pronounced as the difference between the edge lengths of the large and small squares increases, or equivalently, as the fundamental frequency of the pattern decreases. Second, Figure 16b shows that the pattern having a fundamental frequency of $4 \mathrm{cpd}$ should be easiest to segregate, and the patterns having lower and higher fundamental frequencies should be more difficult to segregate. Figure 15b shows, however, that the functions describing the subjects' segregation ratings cross.

These discrepancies suggest that the simple model does not make sufficient use of information in the channels sensitive to the higher harmonics of the pattern. The channel corresponding to the fundamental frequency of the pattern is unable to separate contrast information from size information and is, therefore, unable to yield any infor mation about size differences between the elements. The channels tuned to frequencies higher than the fundamental frequency are increasingly able to transmit information about the sizes of the elements; the highest frequency channels only respond at the edges of the elements and thus could signal size very accurately. It is possible that the outputs of channels tuned to frequencies much higher than the fundamental frequency provide information that is used by the segregation process, in the form of size differences (differences in the lengths of edges) between the elements. The detection of size diferences per se between the elements is, however, not sufficient information for segregation because size differences occur in all of the regions of the pattern. What is important for segregation is the arrangement of the different-sized elements, and this information is not captured by the simple model.

If information in the higher harmonics is used by the segregation process, it must be used in a way that is different from the way information is captured by our simple model. One possibility is that grouping processes, such as those proposed by Beck et al. (1983), operate on feature differences (such as edge length) that are apparent in the outputs of the high spatial-frequency channels. These processes must use explicit coding of the positions and arrangements of these features, linking them into long lines in the top and bottom regions of the texture pattern. The long lines are emergent features that segregate the center regions from the top and bottom regions.

\section{Complex Channels Model}

Another way in which the information in the higher harmonics may be used involves a more complicated spatialfrequency channel. As illustrated in Figure 18, each such channel contains three stages: linear filtering, followed by a nonlinear function such as a rectification, followed by a second linear filter. The filters in both stages are selective for spatial frequency and orientation. A large number of such channels tuned to various spatial frequencies and orientations may exist. The qualitative arguments below require channels in which the first-stage filters are

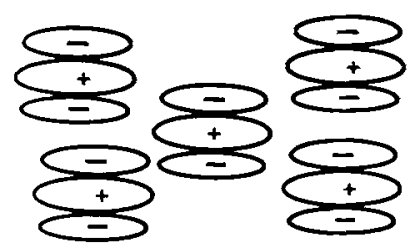

\author{
First Stage \\ A linear filter, \\ e.g. horizontal and of \\ high spatial frequency
}

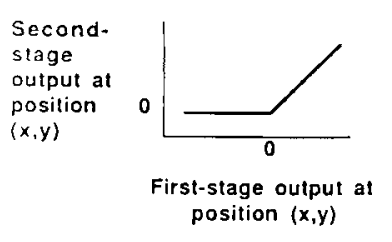

\section{Second Stage}

A point-by-point nonlinearity, dramatic near zero, e.g. a rectification

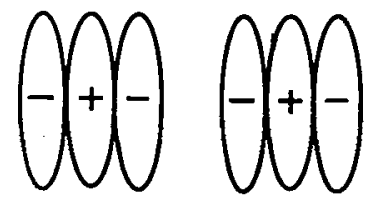

Third stage

A linear filter, e.g. vertical and of low spatial frequency

\section{A Complex Channel}

Figure 18. One channel of the complex model.

sensitive to higher spatial frequencies than the secondstage filters. Whether other relationships are also necessary and/or permissible is not clear. We call these channels "complex" to distinguish them from the earlier simple channels, because this kind of channel is similar to current models of complex cells (e.g., Hochstein \& Spitzer, 1985; Spitzer \& Hochstein, 1985a, 1985b). Measures of pooled second-stage filter activity (analogous to that in the simple model) are taken to be the predictor of perceived texture segregation.

Using complex channels to overcome prediction failures of linear, simple channels was suggest by Robson (1980). Others have been exploring similar ideas (Chubb \& Sperling, 1988; Grossberg \& Mingolla, 1985). Grossberg and Mingolla have proposed a model containing the three stages of the complex model plus additional processes, and have suggested that their model accounts for perceived segregation in patterns such as ours. Their demonstrations, however, involve only filterings sensitive to the higher harmonics of the pattern (small receptive fields relative to the pattern periodicity). On the basis of our findings, we believe that it is unlikely that higher harmonic information is the major determinant of perceived segregation. The tradeoff between area and contrast suggests that the low frequencies matching the period of the pattern are important in texture segregation.

The discrepancies discussed above between the simple model predictions and our experimental results seem to be explainable by the complex channels model. Let us ex- 
amine in some detail how this complex channels model might explain the finding that the greater the size difference between squares, the greater the minimal segregation rating (the shallower the U-shaped functions of Figures $6 a-6 c$ and 9a). Consider a complex channel in which the first filtering is sensitive to a very high spatial frequency and the second filtering (after the nonlinearity) is sensitive to a spatial frequency near the fundamental frequency of the pattern. Such a complex channel can respond to what might be called "low spatial-frequency patterns of high spatial-frequency elements." For our example, we will consider the channel in which both stages are sensitive to vertical orientations. The first highfrequency filtering extracts the edges of the squares as illustrated by the narrow dark and light bands in the right panels of Figures 2 and 3 . The nonlinearity then changes below-zero first-stage outputs (dark bands in Figures 2 and 3) either to zero outputs (if half-wave rectification or something similar is assumed) or to above-zero outputs (if full-wave rectification or something similar is assumed). Since the large squares have longer edges than the small squares, the second-stage filter (which is sensitive to the fundamental frequency of the pattern and to vertical orientations) will have greater outputs in which there are columns of large squares than in which there are columns of small squares, when the contrasts of the squares are equal. Remember that the area of squares increases quadratically with edge length. Therefore, at the contrast ratios at which area $\times$ contrast of the large and small squares are equated, the edge length $x$ contrast of the small square will actually be greater than that of the large square. The amount by which it is greater will be larger for the 16- and 8-pixel case, for example, than for the 16- and 12-pixel case. In general, when both edge lengths and areas count, as in the complex channels model, the edges should attenuate the dip in the U-shaped function more for larger size discrepancies between squares (as long as lower sensitivity to smaller size does not cancel out the greater size difference). This prediction is consistent with the data in Figures 6a-6c and 9a.

In contrast, when the texture elements were lines (see Figure $9 \mathrm{~b}$ ), the minimum texture-segregation ratings were approximately equal and were little affected by the size difference between large and small elements. This also is what would be expected from the complex channels model. When lines (very thin rectangles that always had the same width) are the texture elements, the area increases linearly with edge length. Thus, when the area $x$ contrast of the large and small rectangles are equated (and the first-stage filterings sensitive to the fundamental frequency show little modulated activity), the edge length $X$ contrast is also equated, so the complex channel discussed above also shows little modulated activity. That this difference between the edge and square elements is consistent with expectations from the complex channels model is further reason for thinking that segregation is not due to explicit coding and linking of edges to form emergent features.

The crossover of the functions for different fundamental frequencies in Experiment 4 (Figures $15 \mathrm{~b}$ and 15c) may also be explained by the complex channels model. Consider the 1- and 4-cpd fundamental frequencies. Because the higher harmonics of the 4-cpd patterns fall in a range of low contrast sensitivity, the perceived segregation of these patterns is primarily determined by initial filtering at the fundamental frequency. Thus, when the area $\times$ contrast of the large and small squares is equated, the rated segregation in Figure $15 \mathrm{~b}$ is close to zero. For the 1-cpd patterns, however, the higher harmonics are in a more sensitive range of the contrast-sensitivity function and can contribute to perceived segregation. According to the complex channels model, the influence of the squares' edges (picked up by the initial filtering at the higher harmonic frequencies followed by rectification and refiltering at the fundamental frequency) will attenuate the dip in the U-shaped function. As one moves away from the point of equal areal contrasts, the differences in the outputs of the initial filtering increase more rapidly for the patterns that have a fundamental frequency of $4 \mathrm{cpd}$ than they do for those with a fundamental frequency of $1 \mathrm{cpd}$ because $4 \mathrm{cpd}$ is closer to the optimum of the contrast-sensitivity function. Thus, the curves should cross.

It is interesting to note that a condition in which the area ratio of large and small squares was $4: 1$ while the sizes of the squares varied appears both in the density experiment (Figure 12b) and in the scaling experiment (Figure 15b). In the density experiment, however, the period of the pattern was kept constant, whereas in the scaling experiment, the period increased proportionately to the sizes of the squares. In both cases, the minimum ratings increased as the sizes of the squares (and thus the difference between the sizes) increased. However, the crossovers occurred only when the fundamental frequency changed. This suggests that it is the changing contribution of edges due to their weighting by contrast sensitivity, rather than the smaller size differences between the squares, that produces the crossovers in Figures $15 \mathrm{~b}$ and $15 \mathrm{c}$.

\section{Summary}

A simple spatial-frequency channels model accounts well for many of the effects of changing contrast and spatial variables on perceived texture segregation. There are systematic discrepancies, however, between the quantitative predictions of this model and observers' ratings of perceived texture segregation. A somewhat more complicated spatial-frequency channels model, in which the individual channels contain two stages of linear filtering with a rectification or similar nonlinearity in between, may well account for all the discrepancies. The specific properties of the complex spatial-frequency channels model 
(e.g., the spatial frequency and orientation selectivity at each stage of filtering) remain to be determined by further experimentation and calculation.

\section{REFERENCES}

ADELSON, E. H., \& Bergen, J. R. (1985). Spatiotemporal models for the perception of motion. Journal of the Optical Society of America A, 2, 284-299.

BECK, J. (1972). Similarity grouping and peripheral discriminability under uncertainty. American Joumal of Psychology, 85, 1-19.

BECK, J. (1982). Textural segmentation. In J. Beck (Ed.), Organization and representation in perception (pp. 285-317). Hillsdale, NJ: Erlbaum.

Beck, J. (1983). Textural segmentation, second-order statistics, and textural elements. Biological Cybernetics, 48, 125-130.

Beck, J., Prazdny, K., Rosenfeld, A. (1983). A theory of textural segmentation. In J. Beck, B. Hope, \& A. Rosenfeld (Eds.), Human and machine vision (pp. 1-38). New York: Academic Press.

Beck, J., SuTter, A., * IvRY, R. (1987). Spatial frequency channels and perceptual grouping in texture segregation. Computer Vision. Graphics, \& Image Processing, 37, 299-325.

BERGEN, I. R. (in press). Theories of visual texture perception. In D. Regan (Ed.), Vision and visual dysfunction: Vol. 10b. Spatial vision. New York: Macmillan.

Bergen, J. R., ADELSON, E. H. (1988). Early vision and texture perception. Nature, 333, 363-364.

Bovik, A. C., Clark, M., Geisler, W. S. (1987). Computational texture analysis using localized spatial filtering. Proceedings of the Workshop on Computer Vision (pp. 201-206). Miami Beach: IEEE Computer Society Press.

CAELLI, T. (1982). On discriminating visual textures and images. Perception \& Psychophysics, 31, 149-159.

CAELLI, T. (1985). Three processing characteristics of visual texture segregation. Spatial Vision, 1, 19-30.

CaELL, T. (1988). An adaptive computational model for texture segmentation. IEEE Transactions on Systems, Man, \& Cybernetics, 18, 1.

Chubr, C., \& Sperling, G. (1988). Processing stages in non-Fourier motion perception. Supplement to Investigative Ophthalmology \& Visual Science, 29, 266.

Clark, M., Bovik, A. C., \& Geisler, W. S. (1987). Texture segmentation using a class of narrowband filters. Proceedings of the IEEE International Conference on Acoustics, Speech, \& Signal Processing.

DAUGMAN, J. G. (1985). Uncertainty relation for resolution in space, spatial frequency, and orientation, optimized by two dimensional visual cortical filters. Joumal of the Optical Society of America A, 2, $1160-1169$.

Daugman, J. G. (1987). Image analysis and compact coding by oriented 2D Gabor primitives. S.P.I.E. Proceedings, 758, 19-30.

Daugman, J. G. (1988). Complete discrete 2-D Gabor transforms by neural networks for image analysis and compression. IEEE Transactions on Acoustics, Speech, \& Signal Processing, 36, 1169-1179.

GAGALOWICZ, A. (1981). A new method for texture field synthesis: Some applications to the study of human vision. IEEE Transactions on Pattern Analysis \& Machine Intelligence, 3, 520-533.

GiNsBURG, A. P. (1984). Visual form perception based on biological filtering. In L. Spillman \& B. R. Wooten (Eds.), Sensory experience, adaptation, and perception (pp. 53-72). Hillsdale, NJ: Erlbaum.

GrAHAM, N. (1980). Spatial frequency channels in human vision: Detecting edges without edge-detectors. In C. S. Harris (Ed.), Visual coding and adaptability (pp. 215-262). Hillsdale, NJ: Erlbaum.

Graham, N. (1981). Psychophysics of spatial frequency channels. In M. Kubovy \& J. R. Pomerantz (Eds.), Perceptual organization (pp. 53-72). Hillsdale, NJ: Erlbaum.

GrAHAM, N. (1985). Detection and identification of near-threshold visual patterns. Journal of the Optical Society of America A, 2, 1468-1482.

Graham, N. (1989). Low-level visual processes and texture segregation. Physica Scripta.
Graham, N. (in press). Visual pattern analyzers. New York: Oxford University Press.

Green, B. F., Wolf, A. K., \& White, B. (1959). The detection of statistically defined patterns in a matrix of dots. American Journal of Psychology, 72, 503-520.

Grossberg, S. (1987). Cortical dynamics of three-dimensional form, color, and brightness perception: 1. Monocular theory. Perception \& Psychophysics, 41, 87-116.

Grossberg, S., \& Mingolda, E. (1985). Neural dynamics of perceptual grouping: Textures, boundaries, and emergent segmentations. Perception \& Psychophysics, 38, 141-171.

Hochstein, S., \& Sitzer, H. (1985). One, few, infinity: Linear and nonlinear processing in the visual cortex. In D. Rose \& V. G. Dobson (Eds.), Models of the visual cortex (pp. 341-350). New York: Wiley.

JULESz, B. (1975). Experiments in the visual perception of texture. Scientific American, 232 34-43.

JuLESZ, B. (1981). Textons, the elements of texture perception and their interactions. Nature, 290, 91-97.

Julesz, B. (1986). Texton gradients: The texton theory revisited. Biological Cybermetics, 54, 245-251.

Julesz, B., \& CARLLI, T. (1979). On the limits of Fourier decompositions in visual texture perception. Perception, 8, 69-73.

Julesz, B., GilberT, E. N., VICTOR, J. D. (1978). Visual discrimination of textures with identical third-order statistics. Biological Cybernetics, 31, 137-140.

KleIN, A., \& Tyler, C. W. (1986). Phase discrimination of compound gratings: Generalized autocorrelation analysis. Journal of the Optical Society of America A, 3, 868-879.

LANDY, M. S., \& BERGEN, J. R. (1988). Texture segregation by multiresolution energy or by structure gradient? (Paper presented at the Annual Meeting of the Optical Society of America.) Technical Digest Series, 11, 162.

MARR, D. (1976). Early processing of visual information. Philosophical Transactions of the Royal Society, London, B275, 483-524.

Robson, J. G. (1980). Neural images: The physiological basis of spatial vision. In C. S. Harris (Ed.), Visual coding and adaptability (pp. 177-214). Hillsdale, NJ: Erlbaum.

ShAPLEY, R., ENROTh-CUGELL, C. (1985). Visual adaptation and retinal gain controls. In N. N. Osborne \& G. J. Chader (Eds.), Progress in retinal research (Vol. 3, pp. 263-346). New York: Pergamon.

SPITZER, H., HochsteIN, S. (1985a). A complex-cell receptive-field model. Journal of Neurophysiology, 53, 1266-1286.

SPITZER, H., \& Hochstein, S. (1985b). Simple- and complex-cell response dependences on stimulation parameters. Joumal of Neurophysiology, 53, 1244-1265.

SUTTER, A. (1987). The interaction of size and contrast in perceived texture segregation: A spatial frequency analysis. Unpublished doctoral dissertation, University of Oregon, Eugene.

SutTer, A., Graham, N., \& Beck, J. (1988). Spatial frequency analyses of texture segregation. Supplement to Investigative Ophthalmology \& Visual Science, 29, 399.

TURNER, M. R. (1986). Texture discrimination by Gabor functions. Biological Cybemetics, 55, 71-82.

Victor, J. D., \& BrodiE, S. E. (1978). Discriminable textures with identical Buffon-needle statistics. Biological Cybermetics, 31, 231-234.

WATSON, A. B. (1983). Detection and recognition of simple spatial forms. In O. J. Braddick \& A. C. Sleigh (Eds.), Physiological and biological preprocessing of images (pp. 110-114). New York: Springer-Verlag.

WERTHEIMER, M. (1923). Untersuchungen zur Lehre von der Gestalt II. Psychologische Forschung, 4, 301-350.

\section{NOTES}

1. Contrast has been defined in a number of ways. Beck et al. (1987) defined contrast as the difference between the luminance of a square and the average luminance of a pattern, divided by the average luminance. 
The contrast ratio of two squares was defined as the contrast of the highcontrast squares, as defined above, divided by the contrast of the lowcontrast squares. It should be noted that the effects involving the interaction of area ratio and contrast in the Beck et al. (1987) experiments are unaffected by whether the average luminance or the background luminance is used to calculate the contrast ratio. We use the background luminance in our contrast calculations.

2. Fundamental frequency of the pattern is defined as the reciprocal of the period of the checked region. We have taken the period to be equal to the number of pixels contained in two rows (or two columns) of elements, plus two interrow spaces. The patterns presented in Figures 1-3 had a period of 56 pixels, which translates to approximately $1 \mathrm{cpd}$.

3. Note that the 4-pixel separation is actually two, rather 1.75 times, the width of the 2-pixel square. The 4-pixel square in this condition was, conservatively, the nearest whole number of pixels to the (impossible) 3.5-pixel separation that would have yielded the 1.75 ratio.

4. The ordering of the prediction curves in Figure 16 is a direct result of our choice of contrast-sensitivity function $S_{\text {obs }}\left(f_{1}, A_{j}\right)$, which peaks at $5.6 \mathrm{cpd}$ (and which indeed determined that choice). To a very good approximation, changing the contrast-sensitivity function would only change the relative vertical positions of the curves for different fundamental frequencies in Figure 16. It would not change their shapes (except in very minor ways) and, therefore, would not improve the model by allowing it to predict the crossover in the observed curves in Figures $15 \mathrm{~b}$ and $15 \mathrm{c}$.

\section{APPENDIX \\ The Two-Dimensional Gabor Filter}

The two-dimensional Gabor Function used in the spatialfiltering model is given by the equation

$$
\begin{gathered}
w(x, y)=m \cdot e^{-\ln 2\left[\left(x-x_{0}\right) / w_{x}\right]^{2}} \cdot \cos \left[2 \pi f\left(x-x_{0}\right)+\theta\right] \\
e^{-\ln 2\left[\left(y-y_{0}\right) / w_{y}\right]^{2}},
\end{gathered}
$$

where $\left(x_{0}, y_{0}\right)$ is the center of the weighting function (receptive field); $f$ is the spatial frequency expressed in cycles/degree of the weighting function (i.e., the reciprocal of the width of an excitatory region plus an inhibitory region); $w_{x}$ and $w_{y}$ give the full bandwidth at half-peak amplitude of the Gaussian envelopes of the Gabor functions perpendicular and parallel to the orientation of the field; $\theta$ gives the symmetry of the field $(0$ giving even symmetry); and $m$ gives the height, or peak amplitude, of the weighting function. Although not explicitly represented in the above equation, the orientation of the filter can be changed by rotating the $x$ and $y$ coordinates.

The calculations were done using Gabor functions with parameters modeled after those of Watson (1983), although Watson's model is more complete in two respects. We used fields with only even symmetry and did not incorporate the decrease in acuity occurring with retinal eccentricity. $w_{x}$ and $w_{y}$ (the width of the field in both directions) were set equal, producing an orientation half-amplitude full bandwidth of $38^{\circ}$ of rotation. The width of the field perpendicular to the orientation direction was chosen so that the spatial-frequency half-amplitude full bandwidth was one octave $\left(w_{x}=w_{y}=2 / 3 f\right)$.

To filter the stimulus patterns used in Experiments 1, 2, and 4 , those patterns were reduced in size by one half, and twodimensional fast Fourier transforms were done over $256 \times 256$ pixels instead of $512 \times 512$. The Fourier transforms of Gabor functions are just Gaussians centered at the appropriate (twodimensional) spatial frequency (the spatial frequency and orientation of the weighting function) and these were multiplied by the Fourier transform of the stimuli before doing the inverse Fourier transform to give $O_{i j}(x, y)$.

The contrast-sensitivity function used to weight the filter's outputs for the predictions shown here was given by the equation

$$
S_{\mathrm{obs}}\left(f_{i}, A_{j}\right)=K(f r)^{p} \cdot e^{-f r},
$$

where $K=2.58, r=0.125, p=0.7$, and $f$ is in cycles/degree. This contrast-sensitivity function was the same for all three orientations except that for spatial frequencies below $1 \mathrm{cpd}$; the output of the horizontally oriented filters was suppressed because they produced artifactual differences due to pattern edges.

The displays in Figures 2-5 were normalized so that within any particular figure, the smallest output produced the lowest, and the largest output produced the highest, possible output on the display screen. An output of zero produced a gray in Figures 4 and 5 that is the same as the borders around the patches.

(Manuscript received August 26, 1988; revision accepted for publication March 23, 1989.) 